\title{
X-RAY OBSERVATIONS OF THE YOUNG PULSAR J1357-6429 AND ITS PULSAR WIND NEBULA
}

\author{
Chulhoon Chang $^{1}$, George G. Pavlov ${ }^{1,2}$, Oleg Kargaltsev ${ }^{3}$, and Yurit A. Shibanov 2,4 \\ ${ }^{1}$ Department of Astronomy \& Astrophysics, Pennsylvania State University, PA 16802, USA; chchang@astro.psu.edu, pavlov@astro.psu.edu \\ ${ }^{2}$ St.-Petersburg State Polytechnical University, St.-Petersburg 195251, Russia \\ ${ }^{3}$ Department of Astronomy, University of Florida, FL 32611, USA; oyk100@astro.ufl.edu \\ ${ }^{4}$ Ioffe Physico-Technical Institute, St.-Petersburg 194021, Russia; shib@astro.ioffe.rssi.ru \\ Received 2011 July 5; accepted 2011 September 15; published 2011 December 15
}

\begin{abstract}
We observed the young pulsar J1357-6429 with the Chandra and XMM-Newton observatories. The pulsar spectrum fits well a combination of an absorbed power-law model $(\Gamma=1.7 \pm 0.6)$ and a blackbody model $\left(k T=140_{-40}^{+60} \mathrm{eV}\right.$, $R \sim 2 \mathrm{~km}$ at the distance of $2.5 \mathrm{kpc}$ ). Strong pulsations with pulsed fraction of $42 \% \pm 5 \%$, apparently associated with the thermal component, were detected in $0.3-1.1 \mathrm{keV}$. Surprisingly, the pulsed fraction at higher energies, $1.1-10 \mathrm{keV}$, appears to be smaller, $23 \% \pm 4 \%$. The small emitting area of the thermal component either corresponds to a hotter fraction of the neutron star surface or indicates inapplicability of the simplistic blackbody description. The X-ray images also reveal a pulsar wind nebula (PWN) with complex, asymmetric morphology comprised of a brighter, compact PWN surrounded by the fainter, much more extended PWN whose spectral slopes are $\Gamma=1.3 \pm 0.3$ and $\Gamma=1.7 \pm 0.2$, respectively. The extended PWN with the observed flux of $\sim 7.5 \times 10^{-13} \mathrm{erg} \mathrm{s}^{-1} \mathrm{~cm}^{-2}$ is a factor of 10 more luminous then the compact PWN. The pulsar and its PWN are located close to the center of the extended TeV source HESS J1356-645, which strongly suggests that the very high energy emission is powered by electrons injected by the pulsar long ago. The $\mathrm{X}$-ray to TeV flux ratio, $\sim 0.1$, is similar to those of other relic PWNe. We found no other viable candidates to power the $\mathrm{TeV}$ source. A region of diffuse radio emission, offset from the pulsar toward the center of the TeV source, could be synchrotron emission from the same relic PWN rather than from the supernova remnant.
\end{abstract}

Key words: ISM: individual objects (HESS J1356-645, G309.8-2.6) - pulsars: individual (PSR J1357-6429) X-rays: individual (CXOU J135605.9-642909, 1RXS J135605.5-642902)

Online-only material: color figures

\section{INTRODUCTION}

Thanks to their rich observational manifestations, young energetic pulsars are among the most attractive targets for the Chandra and XMM-Newton observatories. These manifestations include thermal emission from the neutron star (NS) surface, non-thermal emission from the pulsar magnetosphere, and a pulsar wind nebula (PWN) produced by the interaction of the pulsar wind with the ambient medium. The magnetospheric and thermal emission can differ in their relative strengths and exhibit distinct spectra and pulse profiles. The magnetospheric emission usually has power-law (PL) spectrum and is strongly pulsed. Detailed studies of several bright young pulsars indicate a nonuniform NS surface temperature distribution with cool $\left(T_{C} \lesssim 100 \mathrm{eV}\right)$ and hot $\left(T_{H} \sim 0.1-0.3 \mathrm{keV}\right)$ thermal components, which are believed to be emitted from the bulk of the NS surface and polar caps, respectively (e.g., Pavlov et al. 2001; Zavlin \& Pavlov 2004; de Luca et al. 2005; Kargaltsev et al. $2005)$. Since the sample of young pulsars with high-quality $\mathrm{X}$-ray spectra is still very small, further observations of young pulsars must be carried out to learn about the early epoch of NS cooling and test magnetospheric emission models.

In addition to X-ray emission from the NS surface and magnetosphere, a significant amount of non-thermal emission is radiated from the PWN. PWNe display various shapes and structures, including (but not limited to) jets and tori, or cometary shaped tails (Gaensler \& Slane 2006). Observations with high angular resolution are particularly useful since they resolve the extended PWN emission, seen downstream of the termination shock (TS), from the magnetospheric non-thermal emission. Comparing properties of these two components, one can learn about the physical processes responsible for an efficient but as yet unknown particle acceleration mechanism energizing PWNe.

Although more than $\sim 70$ PWNe have been observed with Chandra and XMM-Newton to date (Kargaltsev \& Pavlov 2008, 2010, hereafter KP08 and KP10), the existing models (Kennel \& Coroniti 1984; Komissarov \& Lyubarsky 2004; van der Swaluw 2005; Bucciantini et al. 2005) still cannot explain some of the observed complex morphologies. Pulsars of similar ages and spin-down luminosities can have PWNe with quite different properties. As of now, it is not clear whether these differences can be solely attributed to different properties of the surrounding interstellar medium (ISM) or if different intrinsic properties of pulsars also play an important role. A larger sample of wellresolved X-ray PWNe associated with pulsars having different intrinsic properties and residing in different environments is needed to further advance our understanding of pulsar winds.

The young pulsar PSR J1357-6429 (hereafter J1357; $P=$ $166 \mathrm{~ms}, \dot{E}=3.1 \times 10^{36} \mathrm{erg} \mathrm{s}^{-1}$, surface magnetic field $B=7.8 \times$ $\left.10^{12} \mathrm{G}\right)$ was discovered in the Parkes Multibeam Pulsar Survey by Camilo et al. (2004). The spin-down age, $\tau \equiv P /(2 \dot{P})=$ $7.3 \mathrm{kyr}$, indicates that $\mathrm{J} 1357$ is one of the youngest pulsars known. The distance of $2.5 \mathrm{kpc}$, estimated from the pulsar's dispersion measure $\left(\mathrm{DM}=127.2 \mathrm{~cm}^{-3} \mathrm{pc}\right)$ and the Galactic free electron density model (Cordes \& Lazio 2002), implies a spin-down flux $\dot{E} /\left(4 \pi d^{2}\right)=1.2 \times 10^{-9} \mathrm{erg} \mathrm{s}^{-1} \mathrm{~cm}^{-2}$.

The pulsar is located within the TeV source HESS J1356-645 (hereafter HESS J1356; Renaud et al. 2008; Abramowski et al. 2011). The extended TeV emission is detected up to $12^{\prime}$ from the peak of the TeV surface brightness, located at R.A. $=13^{\mathrm{h}} 56^{\mathrm{m}}$, decl. $=-64^{\circ} 30^{\prime}$. Given the relatively small angular separation, 
$\sim 7^{\prime}$, between J1357 and the center of the HESS source, and the $\mathrm{TeV}$ luminosity, $L_{\mathrm{TeV}} \sim 6 \times 10^{33} \mathrm{erg} \mathrm{s}^{-1} \sim 0.002 \dot{E}$ (at the $2.5 \mathrm{kpc}$ distance), it seems plausible that the HESS source could be powered by this pulsar. The TeV spectrum of HESS J1356 fits PL with the photon index $\Gamma_{\mathrm{TeV}} \approx 2.2$, without a cutoff at high energies. A possible detection of $\mathrm{J} 1357$ with AGILE at lower $\gamma$-ray energies $(100 \mathrm{MeV}-10 \mathrm{GeV})$ was reported by Pellizzoni et al. (2009). ${ }^{5}$ No optical counterpart to the pulsar or its PWN has been detected yet (Mignani et al. 2011).

At radio frequencies, Duncan et al. (1997) reported the extended emission from the supernova remnant (SNR) candidate G309.8-2.6, which is spatially coincident with HESS J1356. The extent of the radio emission appears to be smaller than that of the $\mathrm{TeV}$ source.

J1357 has been previously observed with XMM-Newton (2005 August 5; 15 ks; PI: F. Camilo) and Chandra HRC-S (2005 November 18 and 19; 16 and 17 ks; PI: M. Mendez, observer L. Kuiper). The analysis of those data was reported by Zavlin (2007) and Esposito et al. (2007). ${ }^{6}$ These authors found that the pulsar spectrum likely consists of thermal and non-thermal components. Zavlin (2007) found pulsations with the radio pulsar period and pulsed fraction of $63 \% \pm 15 \%$, while Esposito et al. (2007) reported only an upper limit of $30 \%$ on modulation amplitude. Zavlin (2007) also reported a tail-like extended emission in the HRC-S image, with a luminosity of $2.5 \times 10^{31} \mathrm{erg} \mathrm{s}^{-1}$ (for $d=2.5 \mathrm{kpc}$ ), while Esposito et al. (2007) concluded that the surface brightness distribution around the pulsar "is consistent with that from a point source" and estimated a $2-10 \mathrm{keV}$ upper limit of $3 \times 10^{31} \mathrm{erg} \mathrm{s}^{-1}$ on the PWN luminosity. To resolve these controversies, reliably disentangle various emission components, and study the relation between the pulsar and HESS J1356, we have carried out deeper observations with the Chandra ACIS and XMM-Newton EPIC detectors.

The details of the observations and data analysis are presented in Section 2. We discuss possible interpretations of the PWN morphology, describe inferences from the pulsar spectrum, and speculate on the nature of HESS J1356 and its relation to the other sources in the field in Section 3.

\section{OBSERVATIONS AND DATA ANALYSIS}

We observed $\mathrm{J} 1357$ for $60 \mathrm{ks}$ with the ACIS detector on board Chandra on 2009 October 8 (ObsID 10880). The target was imaged on the $\mathrm{I} 3$ chip, $\approx 30^{\prime \prime}$ from the aim point. The other chips operated during the observation were I0, I1, I2, S2, and S3. The observation was carried out in timed exposure mode and telemetered in Very Faint format. The detector was operated in full frame mode, which provides time resolution $3.24 \mathrm{~s}$. The useful effective exposure time (live time) is $59.22 \mathrm{ks}$. There were no significant particle background flares during the observation. The data were reduced and analyzed with the Chandra Interactive Analysis Observations (CIAO) package (ver. 4.2), with CALDB 4.1.4. We used Chandra Ray Tracer

\footnotetext{
5 After our paper was submitted, Lemoine-Goumard et al. (2011) reported results of observations of $\mathrm{J} 1357$ with Fermi LAT. They detected $\gamma$-ray pulsations and measured the photon index $\Gamma_{\mathrm{GeV}} \approx 1.5$, found an exponential cutoff at $\approx 0.8 \mathrm{GeV}$, and estimated the luminosity $L_{\mathrm{GeV}} \approx 2 \times 10^{34} \mathrm{erg} \mathrm{s}^{-1}$, for $E>0.1 \mathrm{GeV}$.

6 It should be noted that first report on the Chandra results was published in "Chandra News" (2007 March, Issue number 14, pp. 12-13 (see

http://cxc.harvard.edu/newsletters/news_14/newsletter14.html) by R. Kraft and A. Kenter). In particular, evidence for the presence of extended emission $(\mathrm{PWN})$ and the detection of pulsed signal (pulsed fraction $40 \% \pm 12 \%$ ) were reported in that publication.
}

$(\mathrm{ChaRT})^{7}$ and MARX software ${ }^{8}$ for image analysis and XSPEC (ver. 12.5.0) for spectral analysis.

J1357 was also observed with the XMM-Newton EPIC MOS1, MOS2, and PN detectors on 2009 August 14 and 15 (ObsID 0603280101). The two MOS detectors were operated in Full Window mode with a time resolution $2.6 \mathrm{~s}$, while the PN detector was in Small Window mode, which provides $6 \mathrm{~ms}$ time resolution (at the expense of reduced efficiency, $\approx 71 \%$ ). The observation was processed with the XMM-Newton Science Analysis Software (SAS; ver. 9.0.0). The total live times of observations were $78.1 \mathrm{ks}$ and $55.5 \mathrm{ks}$ for EPIC MOS and $\mathrm{PN}$, respectively. However, the data were contaminated by strong flares, which exceeded the quiescent count rate ( 0.1 counts $\mathrm{s}^{-1}$ for MOS and 0.2 counts $\mathrm{s}^{-1}$ for $\left.\mathrm{PN}\right)$ by a factor of up to eight. After excluding contaminated data, the useful scientific exposure times are 62.2 and $44.6 \mathrm{ks}$ for MOS and $\mathrm{PN}$, respectively. The medium filter was used for all the EPIC detectors. The data were filtered to allow only standard event grades (patterns $\leqslant 12$ for MOS and $\leqslant 4$ for $\mathrm{PN}$ ).

\subsection{Images \\ 2.1.1. Chandra}

To produce Chandra images of the pulsar and its vicinity at subpixel resolution, we removed the pipeline pixel randomization and applied the subpixelization tool which improves quality of the images using grades of split-pixel events (Mori et al. 2001; Tsunemi et al. 2001). Figure 1 shows the Chandra image of the region around $\mathrm{J} 1357$ and the extraction regions on the ACIS-I3 chip used for the image and spectral analysis in the $0.3-8 \mathrm{keV}$ energy band. In the image we see a bright pointlike source surrounded by diffuse emission. The position of the center of the brightest pixel is R.A. $=13^{\mathrm{h}} 57^{\mathrm{m}} 02^{\mathrm{s}} .496$, decl. $=-64^{\circ} 29^{\prime} 30^{\prime} \cdot 06$ (J2000.0). The radio pulsar position is R.A. $=13^{\mathrm{h}} 57^{\mathrm{m}} 02 \mathrm{~s} .43(2)$, decl. $=-64^{\circ} 29^{\prime} 30^{\prime}$.2(1) (Camilo et al. 2004). The difference of 0.45 between the X-ray and radio positions is smaller than the error in absolute Chandra astrometry ( 0 '. 6 at the $90 \%$ confidence level ${ }^{9}$ ). We measure the background on the I3 chip in the 50" radius circle centered at about 1.5 southeast from the source. The bright pointlike source contains 371 counts in the $r=1^{\prime \prime}$ aperture, including $12 \%$ contribution from the background and the diffuse emission around the source.

The diffuse emission in the immediate vicinity of the pulsar (region PWN1; shown in the inset in Figure 1), excluding the region of $r=1^{\prime \prime} .5$ circle centered on the pulsar, has 282 total counts in $91 \operatorname{arcsec}^{2}$, including $4.6 \%$ background contribution. The background-subtracted surface brightness within the PWN1 region is $\simeq 2.97 \pm 0.19$ counts $\operatorname{arcsec}^{-2}$. We also define the PWN2 region (see the same inset in Figure 1), which includes the diffuse feature extending northeast of the pulsar and turning northward at $\sim 23^{\prime \prime}$ from the pulsar. This region contains 74 counts in $108 \operatorname{arcsec}^{2}$, including $21 \%$ background contribution. This corresponds to the background-subtracted surface brightness of $\simeq 0.54 \pm 0.08$ counts $\operatorname{arcsec}^{-2}$.

The larger exposure-corrected, binned and smoothed image (Figure 1) shows an even fainter diffuse emission extending $\sim 2.5$ westward and over 1.5 northward. The PWN region on the $I 1$ chip (PWN I1; $\approx 1921 \operatorname{arcsec}^{2}$ ) includes 295 total counts, of which about $70 \%$ comes from the background, estimated from the $150^{\prime \prime}$ radius circle at about $5^{\prime}$ north-northeast of the source

\footnotetext{
7 See http://cxc.harvard.edu/chart/threads/index.html.

8 See http://space.mit.edu/CXC/MARX/.

9 See http://cxc.harvard.edu/cal/ASPECT/celmon/
} 


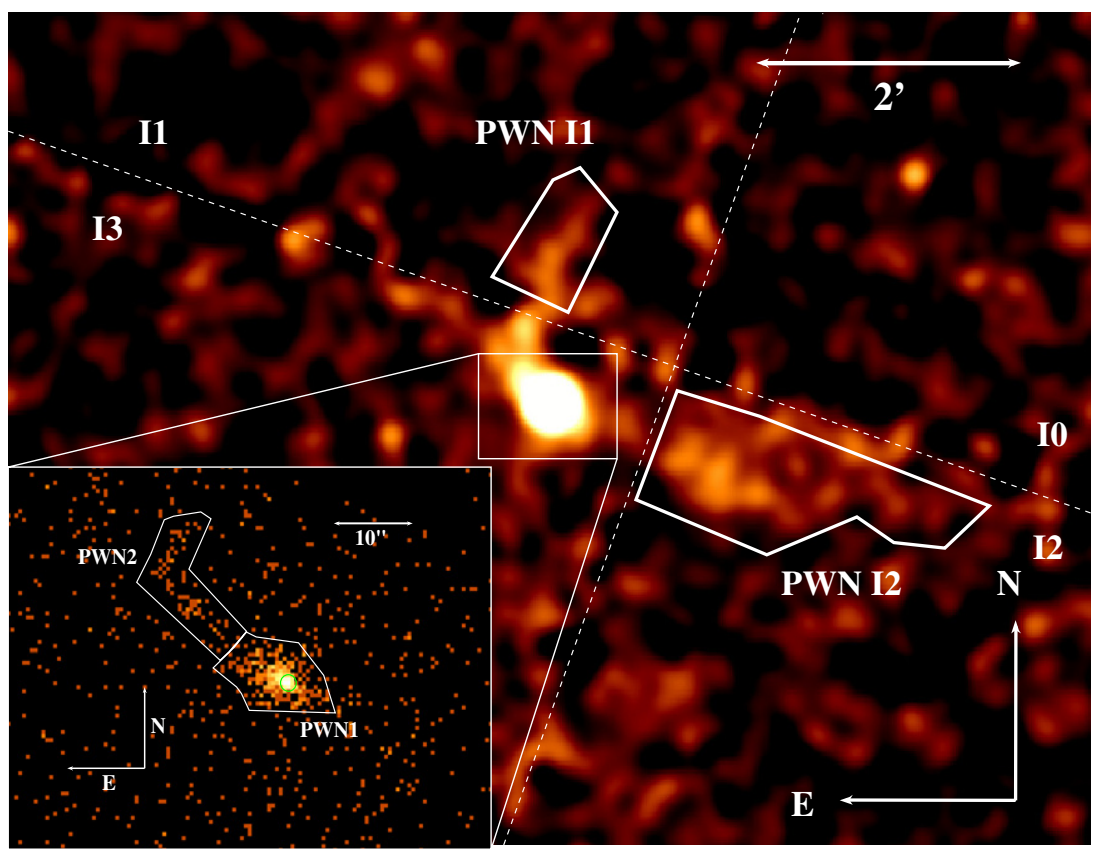

Figure 1. Chandra images of J1357 and its PWN in the 0.3-8.0 keV band. The exposure-corrected larger image is binned to a pixel size of 0 '.98 and then smoothed with the Gaussian kernel of about $3^{\prime \prime}$ radius using the aconvolve script. The dashed lines show the chip boundaries. PWN I1 and PWN I2 are the extraction regions (see the text). The zoomed unsmoothed image in the inset shows the extraction regions for the image and spectral analysis.

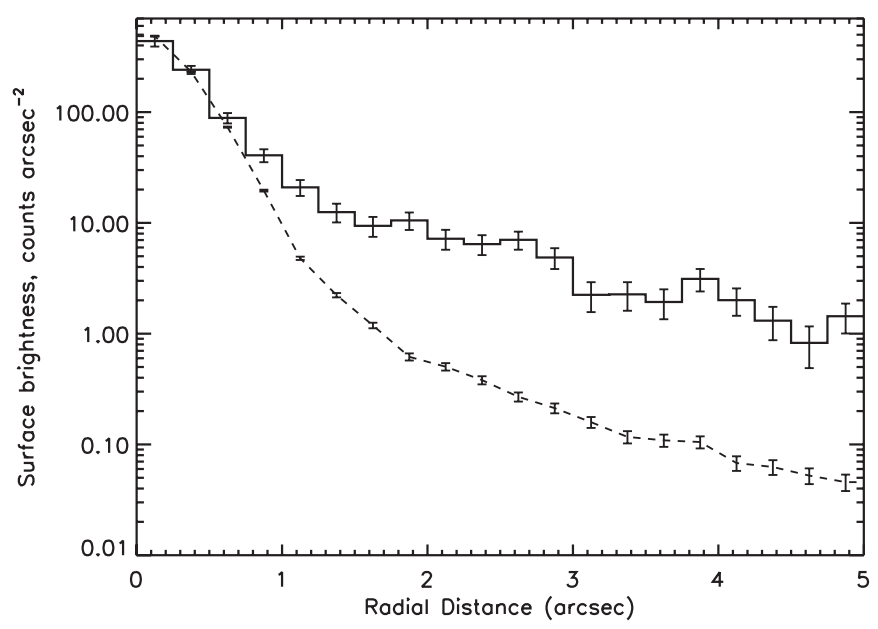

Figure 2. Radial profiles of the observed emission (solid histogram with bin size 0.25 ) around the pulsar and the simulated PSF (dashed line) in the $0.3-8.0 \mathrm{keV}$ energy band. The PWN emission dominates the pulsar PSF at $r>0$ '.75 from the pulsar.

region. The total number of counts in the PWN region on I2 chip (PWN I2; $\approx 6128 \operatorname{arcsec}^{2}$ ) is 1036, including 72.5\% contribution from the background, estimated from the $100^{\prime \prime}$ radius circle located at just south of the source region. The backgroundsubtracted PWN surface brightness within the source regions on I1 and I 2 chips is $0.046 \pm 0.009$ and $0.047 \pm 0.006$ counts $\operatorname{arcsec}^{-2}$, respectively.

The radial profile of the emission centered on the brightest pixel position is plotted in Figure 2, with the simulated pointspread function (PSF) shown for comparison. To simulate the PSF, we used the ChaRT and MARX packages following the standard procedure. To reduce the statistical errors of the simulation, the normalization of the input spectrum was increased by a factor of 100 while running ChaRT. The simulated images were then rescaled back for direct comparison with the data. We have also tried several values of the MARX Dither Blur parameter and found an optimal value of 0 '.25, which minimizes the difference between the simulated PSF and the observed count distribution in the immediate vicinity of the pulsar position. The histogram in Figure 2 shows that the PWN starts dominating the unresolved pulsar emission at $\gtrsim 0$ '.75 from the pulsar.

\subsubsection{XMM-Newton}

Figure 3 shows the combined EPIC MOS1+MOS2 image (in $0.3-10 \mathrm{keV}$ ) and several regions that we use in our analysis. Within the $r=20^{\prime \prime}$ circle centered on the pulsar we find 1900 MOS1+MOS2 counts, of which the background (taken from the $r=100^{\prime \prime}$ circle east of the pulsar) contributes about $15 \%$. The MOS images show the large-scale PWN morphology reminiscent of that seen in the Chandra image (cf. Figure 1). Indeed, the contours from the MOS $1+2 \mathrm{PWN}$ image match the Chandra PWN image quite well (see Figure 4). Since the source is near the chip edge in the PN image, only part of the extended emission can be seen, while the other part is out of the field of view (FOV; see Figure 5). We choose an $r=20^{\prime \prime}$ circle centered on the pulsar in the PN image for the source region, and a $60^{\prime \prime}$ radius circle, centered at about 2.5 south-southwest from the source for the background region. The total number of counts in the source region is 2403 , including $19 \%$ background contribution.

To choose an optimal PWN region in the XMM-Newton MOS and PN images, we simulated the PSF using Quicksim ${ }^{10}$. The simulation shows that an $r=30^{\prime \prime}$ circle around the position of the pulsar contains an $88 \%$ encircled count fraction for the point source. Excluding this circle from the PWN polygon region (see Figure 3) leaves 3446 counts in MOS1+2, including $\sim 60 \%$ background contribution. Although some regions of the PWN are out of the PN Small Window FOV (see Figure 5), we still collected 3771 counts, including $\sim 70 \%$ background contribution.

\footnotetext{
$\overline{10}$ See http://heasarc.gsfc.nasa.gov/docs/xmm/quicksim/quicksim.html.
} 


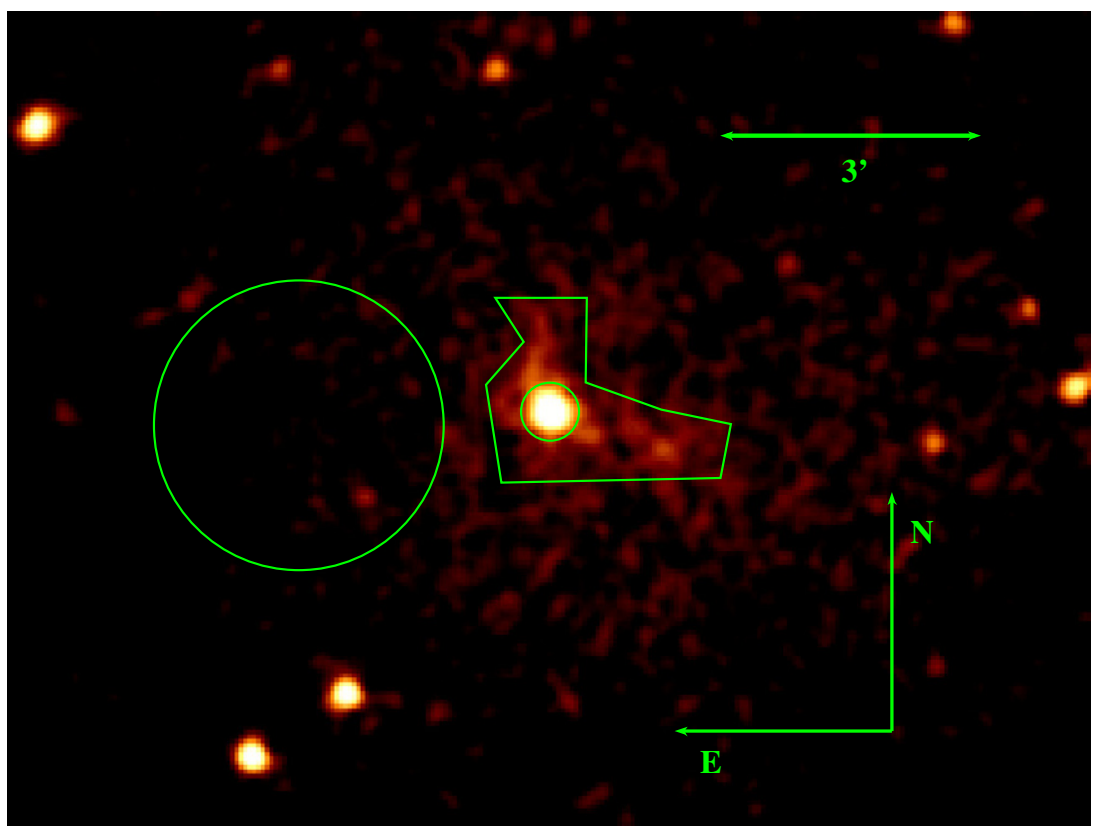

Figure 3. XMM-Newton EPIC MOS1+MOS2 image of J1357 and its PWN in the $0.3-10 \mathrm{keV}$ energy band. The image is binned to a pixel size of 3 " and then smoothed with a Gaussian kernel of 9" radius. Extraction regions $\left(r=20^{\prime \prime}\right.$ circle for the pulsar region, 11,210 $\operatorname{arcsec}^{2}$ polygon for the PWN region, and $r=100^{\prime \prime}$ circle for the background region) for the spectral analysis are also shown.

(A color version of this figure is available in the online journal.)

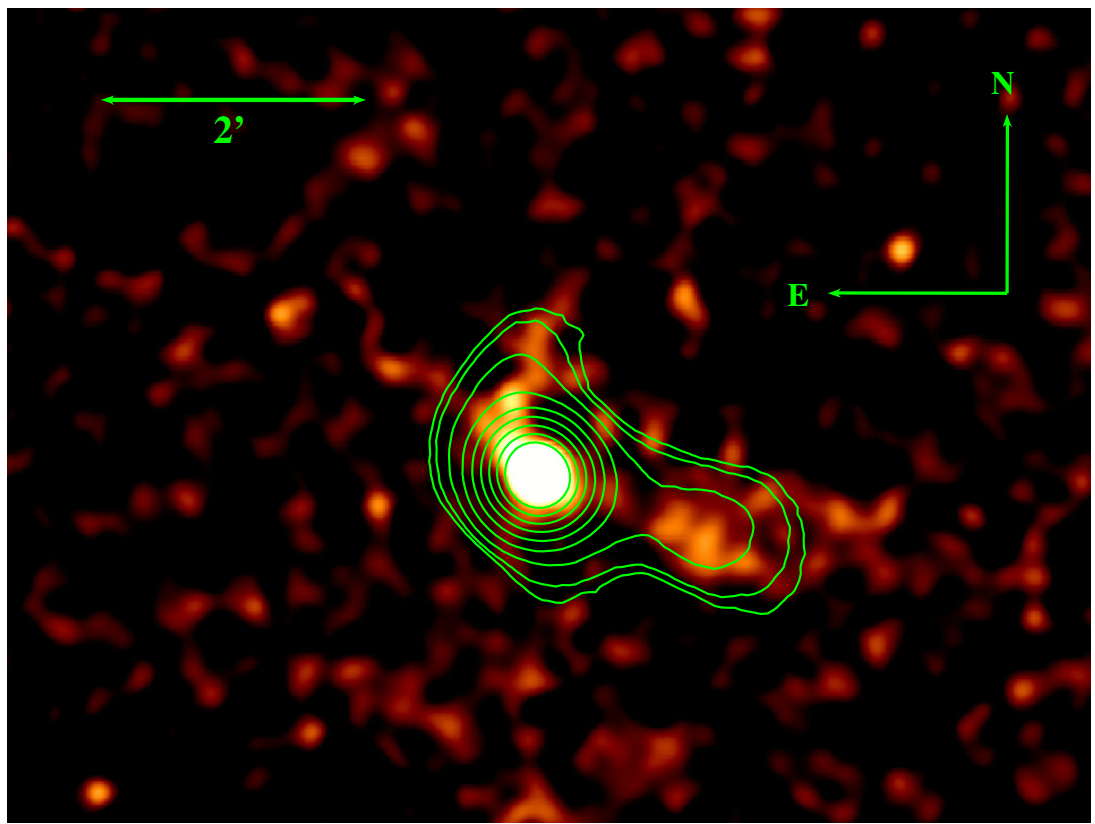

Figure 4. Smoothed Chandra ACIS image from Figure 1 with contours of the XMM-Newton MOS1+2 image overlaid. The contours correspond to the brightness levels of $2.9,3,3.3,4,5,6,7,8$, and 9 counts.

(A color version of this figure is available in the online journal.)

\subsubsection{Other Sources in the Field of Chandra and XMM-Newton}

Panels (a) and (b) of Figure 6 show that J1357 is the brightest extended X-ray source within the imaged part of the HESS J1356 field. Nevertheless, we have examined the other sources in the ACIS FOV. Three brightest sources on the S3 chip southwest of the circle in Figure 6(a) have Two Micron All Sky Survey (2MASS; Skrutskie et al. 2006) counterparts. Most likely, they are stars unrelated to the $\mathrm{TeV}$ source. There are five relatively bright sources inside the circle, which do not have 2MASS counterparts (Sources 1-5 in Figure 6(a)). Four of these sources are seen in both the ACIS and MOS images, while Source 2 fell into the chip gap in MOS. One more source, lacking a 2MASS counterpart, is seen in the MOS images (Source 6 in Figure 6(b)), but it happens to be outside the ACIS FOV. We fitted an absorbed PL model to the spectrum of each of these sources. When the PL model was unacceptable, we fitted the spectrum with the blackbody (BB) model. The spectral properties of the sources are summarized in Table 1 . These sources could be cataclysmic variables or quiescent low-mass X-ray binaries, or they could be active galactic nuclei seen through the Galactic disk. 


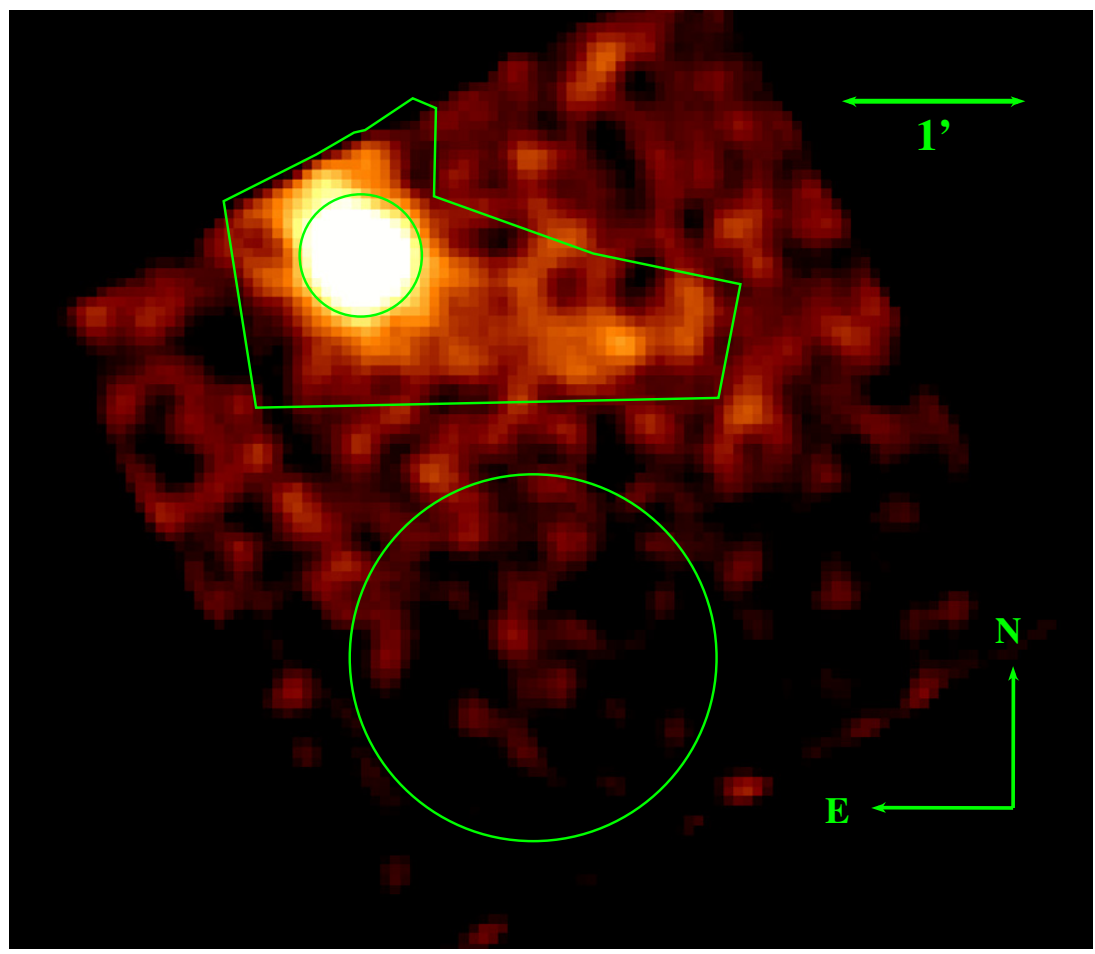

Figure 5. XMM-Newton EPIC PN image is binned to a pixel size of $3^{\prime \prime}$ and then smoothed with a Gaussian kernel about $9^{\prime \prime}$ in radius. The image also shows the $r=20^{\prime \prime}$ circle for the pulsar region, $9159 \operatorname{arcsec}^{2}$ polygon for the PWN region, and $r=60^{\prime \prime}$ circle for the background region for the spectral analysis of J1357 and its PWN in 0.3-10 keV. Unfortunately, the northern part of the PWN emission cannot be seen because the source is near the chip edge.

(A color version of this figure is available in the online journal.)

Table 1

Spectral Fits of the Field Sources in the Chandra and XMM-Newton Images

\begin{tabular}{lcccccccc}
\hline \hline Source & $n_{\mathrm{H}, 21}$ & $\Gamma$ & $\mathcal{N}^{\mathrm{a}}$ & $k T^{\mathrm{b}}$ & $\mathcal{N}_{T}{ }^{\mathrm{c}}$ & $F_{\text {abs }}{ }^{\mathrm{d}}$ & $F_{\text {unabs }}{ }^{\mathrm{e}}$ & $\left(C{\left.\text { or } \chi_{\nu}^{2}\right) / \text { dof }^{\mathrm{f}}}^{\mathrm{f}}\right.$ \\
\hline 1 & $12 \pm 4$ & $1.78_{-0.35}^{+0.39}$ & $4 \pm 5$ & $\ldots$ & $\ldots$ & $14 \pm 1$ & $28_{-6}^{+12}$ & $1.05 / 30$ \\
2 & $3_{-3}^{+4}$ & $\ldots$ & $\ldots$ & $1.6_{-0.3}^{+0.5}$ & $2_{-1}^{+2}$ & $9 \pm 2$ & $10 \pm 2$ & $0.88 / 17$ \\
3 & $3_{-3}^{+4}$ & $\ldots$ & $\ldots$ & $1.1 \pm 2$ & $6_{-3}^{+7}$ & $6 \pm 1$ & $6 \pm 1$ & $0.92 / 16$ \\
4 & $3_{-3}^{+6}$ & $\ldots$ & $\ldots$ & $1.0_{-0.2}^{+0.3}$ & $3_{-2}^{+5}$ & $3.3_{-0.7}^{+0.8}$ & $3.7_{-0.7}^{+0.9}$ & $9.16 / 8$ \\
5 & $3_{-3}^{+4}$ & $1.9_{-0.6}^{+0.8}$ & $9_{-9}^{+19}$ & $\ldots$ & $\ldots$ & $3.0_{-0.8}^{+0.9}$ & $5_{-1}^{+4}$ & $0.98 / 11$ \\
6 & $7 \pm 2$ & $1.54_{-0.22}^{+0.27}$ & $40 \pm 10$ & $\ldots$ & $\ldots$ & $17 \pm 2$ & $25_{-3}^{+5}$ & $0.84 / 39$ \\
\hline
\end{tabular}

Notes. The errors shown represent $90 \%$ confidence intervals.

${ }^{\text {a }}$ PL normalization in units of $10^{-6}$ photons $\mathrm{cm}^{-2} \mathrm{~s}^{-1} \mathrm{keV}^{-1}$ at $1 \mathrm{keV}$.

${ }^{\mathrm{b}} \mathrm{BB}$ temperature in $\mathrm{keV}$.

${ }^{\mathrm{c}} \mathrm{BB}$ normalization $\mathcal{N}_{T}=10^{-3} R_{\mathrm{km}}^{2} / D_{10}^{2}$, where $R_{\mathrm{km}}$ is the source radius in $\mathrm{km}$, and $D_{10}$ is the distance to the source in units of $10 \mathrm{kpc}$.

$\mathrm{d}$ Absorbed flux in the $0.3-8 \mathrm{keV}$, in units of $10^{-14} \mathrm{erg} \mathrm{cm}^{-2} \mathrm{~s}^{-1}$.

${ }^{\mathrm{e}}$ Unabsorbed flux in the $0.3-8 \mathrm{keV}$, in units of $10^{-14} \mathrm{erg} \mathrm{cm}^{-2} \mathrm{~s}^{-1}$.

${ }^{\mathrm{f}}$ Best-fit $C$-statistic value (Source 4 ) or reduced $\chi^{2}$ value (other sources).

Finally, we investigated in detail the relatively bright source on the ACIS I0 chip, CXOU J135605.9-642909 (hereafter CXOU J1356), which is apparently the same source as 1RXS J135605.5-642902. The source is also seen in the EPIC images, but it is near the chip gap in the EPIC MOS1 and MOS2 images. We extracted 443 total counts within the $5^{\prime \prime}$ radius around the brightest pixel of the source from the ACIS image. The spectrum was binned to have a minimum of 25 counts per bin. We did not obtain an adequate fit with a singlecomponent (PL or BB) model (e.g., $n_{\mathrm{H}} \approx 2.0 \times 10^{21} \mathrm{~cm}^{-2}$, $\Gamma \approx 3.4$, and $\chi_{v}^{2}=1.8$ for 14 degrees of freedom (dof) for the PL model). The spectrum fits better the two-component PL+BB model, which gives $n_{\mathrm{H}} \approx 4.3 \times 10^{21} \mathrm{~cm}^{-2}, \Gamma=2.9$, $k T=0.12 \mathrm{keV}$, and $\chi_{v}^{2}=1.1$ for $12 \mathrm{dof}$. The absorbed flux is $6.4 \times 10^{-14} \mathrm{erg} \mathrm{cm}^{-2} \mathrm{~s}^{-1}$ in the $0.3-8 \mathrm{keV}$ band. We found no significant variability in the light curve. We have searched the field at other wavelengths to understand the nature of the source. The nearest optical/IR source was found in the Naval Observatory Merged Astrometric Dataset (NOMAD; Zacharias et al. 2005) and 2MASS catalogs $(B=13.81, V=13.66$, $R=12.69, J=12.199 \pm 0.029, H=11.779 \pm 0.031$, and $K=11.768 \pm 0.030$ ), offset by $\approx 0$ '. 4 from the position of CXOU J1356. The optical-NIR colors show that the source might be a $\mathrm{G}$ or $\mathrm{K}$ star. The estimated X-ray to optical flux ratio is about $4 \times 10^{-3}$, which is similar to a typical value $\left(\sim 10^{-3}\right)$ for a $\mathrm{G}$ or K star (Maccacaro et al. 1988). 

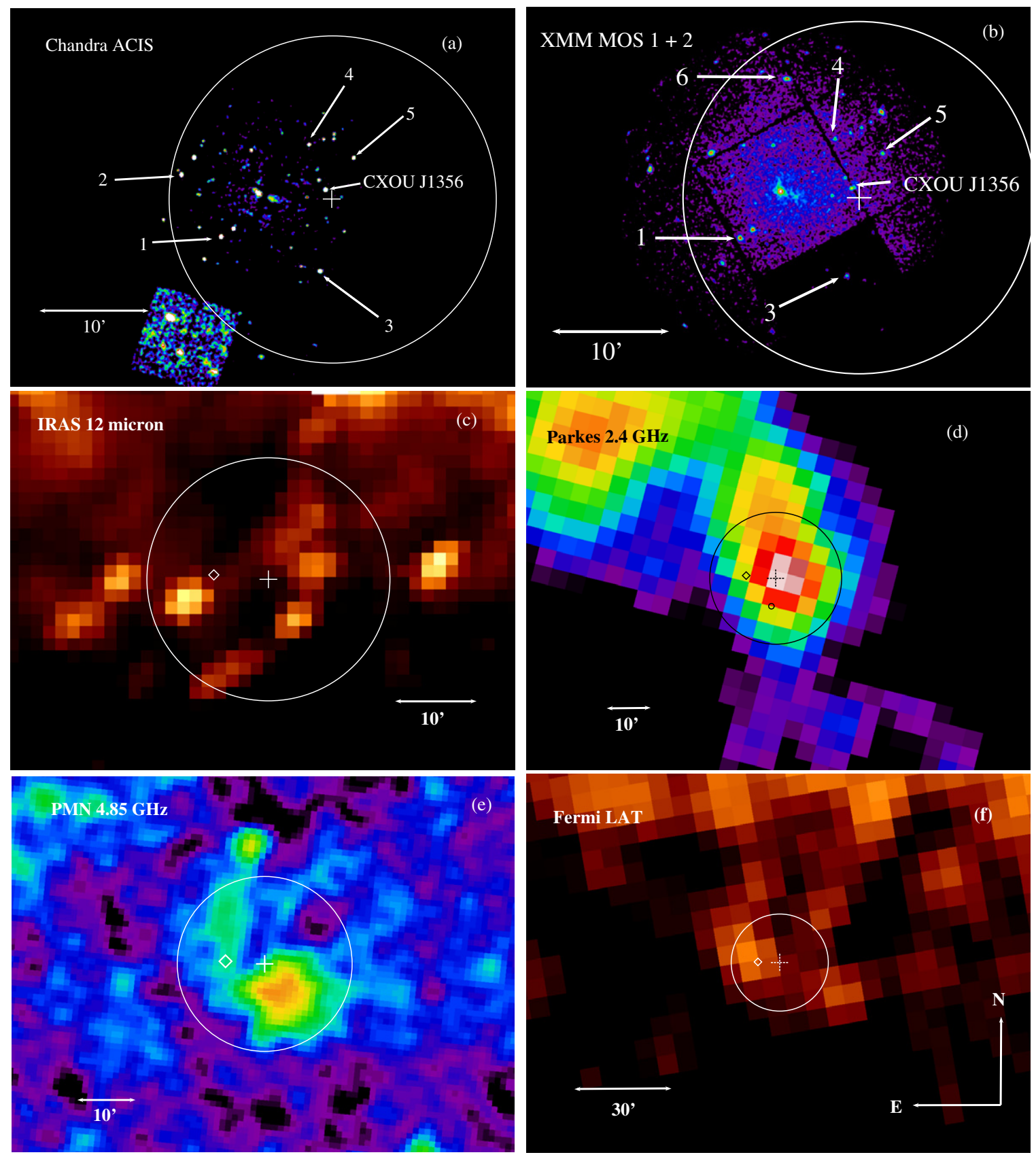

Figure 6. X-ray, IR, and radio images of the J1357 and HESS J1356 field. The cross shows the centroid position of HESS J1356, while the circle (15' radius) indicates the intrinsic (rms) Gaussian width of the source, according to Renaud et al. (2008). The white arrows in panels (a) and (b) indicate CXOU J135605.9-642909 and six other sources (see the text for details). (a) Chandra ACIS image in 0.5-8 keV. (b) XMM MOS1+2 image in the 0.3-10 keV. (c) $12 \mu \mathrm{m} I R A S$ image. (d) $2.4 \mathrm{GHz}$ Parkes image. The small circle located south of the cross point indicates the position of SNR candidate G309.8-2.6. (e) 4.85 GHz PMN image. (f) Fermi LAT image. The diamond in panels (c)-(f) shows the position of J1357.

Although many more sources are seen in the ACIS and MOS images, the low numbers of counts preclude spectral fitting, making it even more difficult to establish the nature of these sources. None of the investigated sources shows evidence for extended emission in X-rays, and there are no compelling reasons to believe that any of them is related to HESS J1356.

\subsubsection{Multiwavelenth Images}

In addition to analyzing the Chandra and XMM-Newton images, we have searched the archival radio and IR data covering the HESS J1356 region. The $12 \mu$ mimage (Figure 6(c)) from IRAS shows several bright sources within the circle. Figure 6(d) shows the $2.4 \mathrm{GHz}$ image from the Parkes survey of the southern Galactic plane. ${ }^{11}$ The extended radio emission has been previously classified as an SNR candidate G309.8-2.6 (Duncan et al. 1997). Another radio image from the Parkes-MIT-NRAO (PMN ${ }^{12}$; Griffith \& Wright 1993) survey at $4.85 \mathrm{GHz}$ also shows an extended emission southeast of HESS J1356 (see Figure 6(e)). The Fermi Large Area Telescope (LAT) image (0.5-100 GeV), shown in Figure 6(f), reveals a faint $\mathrm{GeV}$ emission near the pulsar position. The angular extent of the $\mathrm{GeV}$ excess is consistent with that expected for the unresolved

\footnotetext{
11 see http://www.atnf.csiro.au/research/surveys/2.4Gh_Southern/data.html.
} 12 see http://www.parkes.atnf.csiro.au/observing/databases/pmn/pmn.html. 
point source. No counterparts to J1357 or its X-ray PWN are apparent in the IR and radio images. Deeper observations of this region with higher spatial resolution would be desirable to understand the nature of the extended radio and IR emission.

\subsection{Spectral and Timing Analysis}

\subsubsection{Pulsar Spectrum}

For the spectral analysis of the Chandra observation, we first extracted the pulsar's spectrum from a small circle ( 1 " radius aperture with an $88 \%$ encircled energy fraction) to reduce the contamination from the PWN (see Figure 1). There are 371 total counts in this aperture, of which about $11 \%$ come from the PWN and 1\% from the background. We binned the spectrum with minimum of 10 counts per bin and used the $C$-statistic (Cash 1979) in spectral fitting. The spectrum was first fitted with a single-component model, such as the absorbed PL model and the absorbed BB model, in $0.3-8 \mathrm{keV}$. The PL fit gave $n_{\mathrm{H}, 21} \equiv n_{\mathrm{H}} /\left(10^{21} \mathrm{~cm}^{-2}\right) \approx 1.4$, and $\Gamma \approx 2.4(C=52$ for 34 bins, 31 dof), while the BB was not statistically acceptable ( $C=173$ for 34 bins, 31 dof). Since the PL fit quality was not good, we fit the same spectrum with the absorbed PL+BB model. The much better fit ( $C=28$ for 34 bins for 29 dof) yields $n_{\mathrm{H}, 21} \approx 4.7, \Gamma \approx 1.7, k T \approx 0.14 \mathrm{keV}\left(T \approx 1.6 \times 10^{6} \mathrm{~K}\right)$, and the projected emitting area $\mathcal{A} \approx 13 d_{2.5}^{2} \mathrm{~km}^{2}\left(d_{2.5}=d / 2.5 \mathrm{kpc}\right)$. This temperature and area correspond to the apparent radius $R=\sqrt{\mathcal{A} / \pi} \sim 2 d_{2.5} \mathrm{~km}$ and bolometric luminosity $L_{\mathrm{bol}}=$ $4 \mathcal{A} \sigma T^{4} \sim 2 \times 10^{32} d_{2.5}^{2} \mathrm{erg} \mathrm{s}^{-1}$. The pulsar's observed flux is $F_{\mathrm{psr}}^{\mathrm{abs}}=6.7_{-0.8}^{+0.9} \times 10^{-14} \mathrm{erg} \mathrm{cm}^{-2} \mathrm{~s}^{-1}$ in $0.3-8 \mathrm{keV}$ (corrected for the $88 \%$ encircled energy fraction), while the corresponding unabsorbed flux is $F_{\mathrm{psr}}^{\text {unabs }}=32_{-21}^{+190} \times 10^{-14} \mathrm{erg} \mathrm{cm}^{-2} \mathrm{~s}^{-1}$ in the same energy band. The unabsorbed luminosity is $L_{\mathrm{psr}}=$ $2.4 \times 10^{32} d_{2.5}^{2} \mathrm{erg} \mathrm{s}^{-1}$. The luminosity of the non-thermal (PL) component is $L_{\mathrm{PL}}=5.2_{-1.5}^{+2.9} \times 10^{31} \mathrm{erg} \mathrm{s}^{-1}(\sim 22 \%$ of the total unabsorbed luminosity), while the luminosity of the thermal (BB) component is $L_{\mathrm{BB}}=1.9_{-1.4}^{+14} \times 10^{32} \mathrm{erg} \mathrm{s}^{-1}$. The uncertainties of the fits are reported in Table 2, and the confidence contours are shown in Figures 7 and 8.

Instead of $\mathrm{BB}$, one can use a neutron star atmosphere (NSA) model for the thermal component, which gives a lower (effective) temperature and a larger size of the emitting region (Pavlov et al. 1995). Since the spectrum emerging from a magnetized atmosphere is anisotropic, one should know the geometry of the surface magnetic field (e.g., the angles between the rotation axis, magnetic axis, and line of sight for a dipole field) to fit the spectrum. For a crude estimate, however, one can use the NSA models in XSPEC, which are calculated for emergent flux assuming the magnetic field perpendicular to the surface. We made such an estimate using the hydrogen NSA models for the NS mass $M=1.4 M_{\odot}$, radius $R=10 \mathrm{~km}$, and magnetic field $B=1 \times 10^{13} \mathrm{G}$. If the distance is fixed at $d=2.5 \mathrm{kpc}$, the fit ( $C=29$ for 30 dof) yields $n_{\mathrm{H}, 21}=4.6_{-0.7}^{+0.8}$, $\Gamma=1.45_{-0.60}^{+0.50}, T_{\text {eff }}=0.96_{-0.05}^{+0.04} \mathrm{MK}\left(T_{\text {eff }}^{\infty}=0.74_{-0.04}^{+0.03} \mathrm{MK}\right.$ for the gravitationally redshifted temperature).$^{13}$ It shows that the thermal component can be emitted from a substantial part of the NS surface.

To compare the Chandra ACIS spectrum with those obtained from the XMM-Newton EPIC detector, we extracted the pulsar spectrum from the $20^{\prime \prime}$ radius aperture for MOS and PN and the background spectrum from the $100^{\prime \prime}$ radius for MOS and $60^{\prime \prime}$ radius for PN (see Figures 3 and 5). The spectrum

\footnotetext{
$\overline{13}$ A similar fit was obtained by Lemoine-Goumard et al. (2011)
}
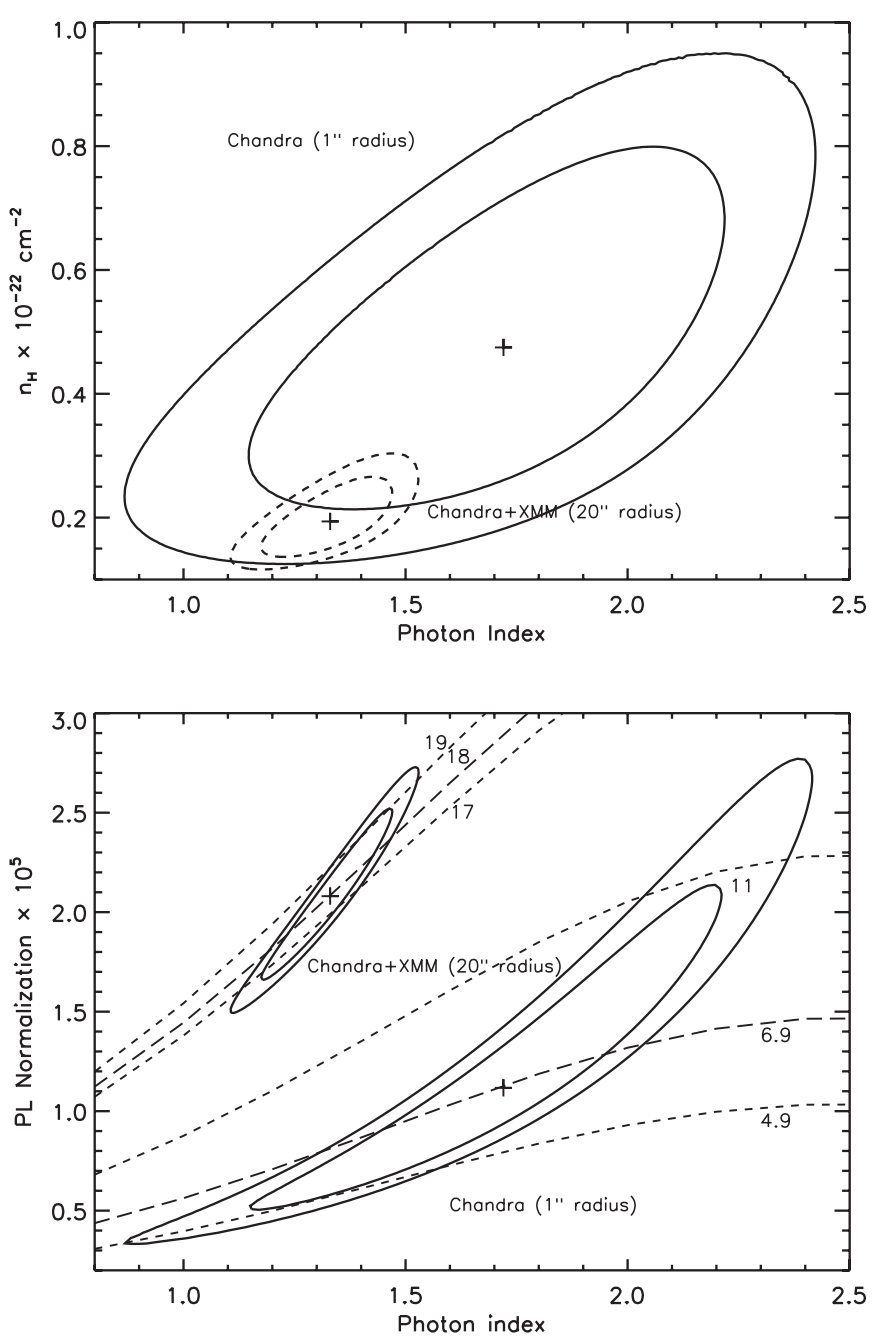

Figure 7. Top: confidence contours (68\% and $90 \%)$ in the $\Gamma-n_{\mathrm{H}}$ plane for the PL+BB fit to the pulsar's spectrum from the Chandra observation ( $1^{\prime \prime}$ extraction radius) and Chandra + XMM-Newton observations (20" extraction radius). Bottom: confidence contours (68\% and $90 \%)$ for the PL component of the PL+BB fit to pulsar's spectrum from the Chandra observation ( 1 " extraction radius) and Chandra $+X M M$-Newton observations (20" extraction radius). The PL normalization is in the units of $10^{-5}$ photons $\mathrm{cm}^{-2} \mathrm{~s}^{-1} \mathrm{keV}^{-1}$ at $1 \mathrm{keV}$. The dashed curves are the lines of constant unabsorbed flux for the PL component in the units of $10^{-14} \mathrm{erg} \mathrm{cm}^{-2} \mathrm{~s}^{-1}$ in $0.5-8 \mathrm{keV}$.

is heavily contaminated by the compact PWN (about $42 \%$ contribution). The spectra were binned with a minimum of 50 counts per bin for each detector. Since the absorbed PL fit was not satisfactory ( $\chi_{v}^{2}=1.94$ for 82 dof), we fitted the absorbed PL+BB model which yields $n_{\mathrm{H}, 21} \approx 2.2, \Gamma \approx 1.35$, $k T \approx 0.18 \mathrm{keV}$, and the projected emitting area $\mathcal{A} \approx 2 d_{2.5}^{2} \mathrm{~km}^{2}$. The BB parameters correspond to the apparent radius $R \sim$ $0.8 d_{2.5} \mathrm{~km}$ and $L_{\text {bol }}=8.6 \times 10^{31} d_{2.5}^{2} \mathrm{erg} \mathrm{s}^{-1}$. Although the best-fit $k T$ and $\mathcal{A}$ from the XMM-Newton observations are different from those observed from the Chandra data, these parameters are consistent within their uncertainties. The observed flux (corrected for the $80 \%$ encircled counts fraction) is $F_{\text {pulsar+PWN }}^{\text {abs }}=2.6 \times 10^{-13} \mathrm{erg} \mathrm{cm}^{-2} \mathrm{~s}^{-1}$, and the corresponding unabsorbed flux is $F_{\text {pulsar+PWN }}^{\text {unabs }}=3.6 \times 10^{-13} \mathrm{erg} \mathrm{cm}^{-2} \mathrm{~s}^{-1}$ in $0.3-10 \mathrm{keV}$. Additional details are provided in Table 2.

The photon index and temperature of the PL+BB model inferred from the XMM-Newton and Chandra observations are consistent within their uncertainties. On the other hand, the absorbed flux estimated from the XMM-Newton data is a factor 


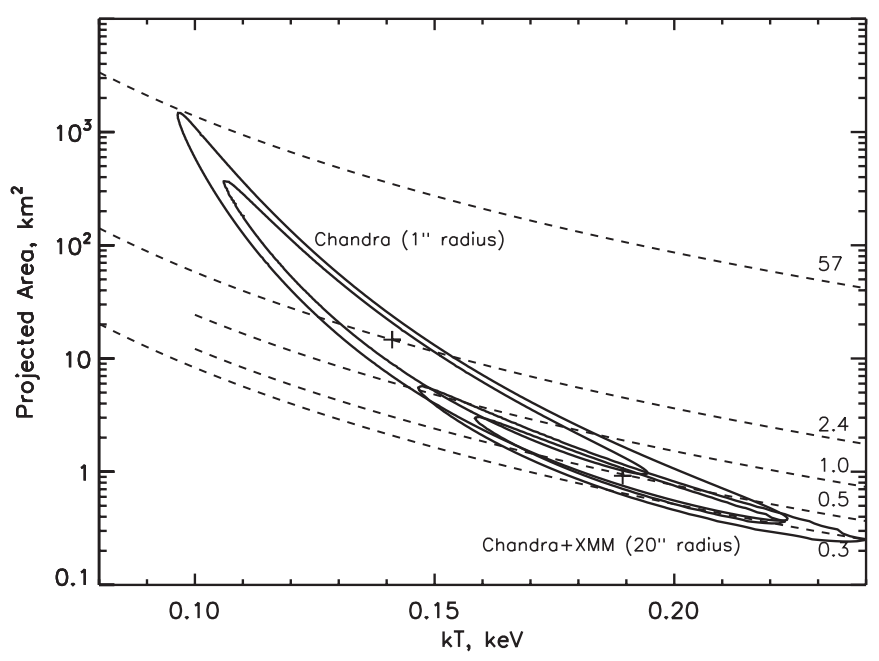

Figure 8. Confidence contours (68\% and 90\%) for the BB parameters of the PL+BB fit to the pulsar's spectrum from the Chandra (1" extraction radius) observation and Chandra $+X M M-N e w t o n$ observations ( $20^{\prime \prime}$ extraction radius). The BB normalization is the projected emitting area $\left(\mathrm{km}^{2}\right)$. The dashed lines show the constant bolometric luminosity in units of $10^{32} \mathrm{erg} \mathrm{s}^{-1}$, assuming $d=2.5 \mathrm{kpc}$.

of three larger than that estimated from the Chandra data due to the larger extraction region (20" radius), chosen for XMMNewton data, which contains a significant PWN contribution. Therefore, we extracted the spectra using the same source region ( $20^{\prime \prime}$ radius) for the Chandra observation to compare with the spectral results obtained from the XMM-Newton observation. The region includes 873 source counts, which is $83 \%$ of the total counts. Now both fits are consistent with each other (see Table 2). Finally, the Chandra (20" radius aperture) and XMMNewton data were fitted jointly with the PL+BB model, which still fits satisfactorily while the uncertainties of the best-fit parameters are significantly reduced compared to the individual fits (see Table 2 and Figure 9).

\subsubsection{PWN Spectrum}

We extracted the ACIS spectra from the PWN1 and PWN2 regions (see Figure 1). For PWN1's spectrum, we excluded the pulsar region (1".5 radius aperture) and used the absorbed PL model in the energy range of $0.3-8 \mathrm{keV}$. The spectrum was binned with minimum of 10 counts per bin. The fit $\left(\chi_{v}^{2}=1.3\right.$ for 24 dof $)$ gives $n_{\mathrm{H}, 21}=3.2_{-1.4}^{+2.3}, \Gamma=1.31_{-0.33}^{+0.37}$,

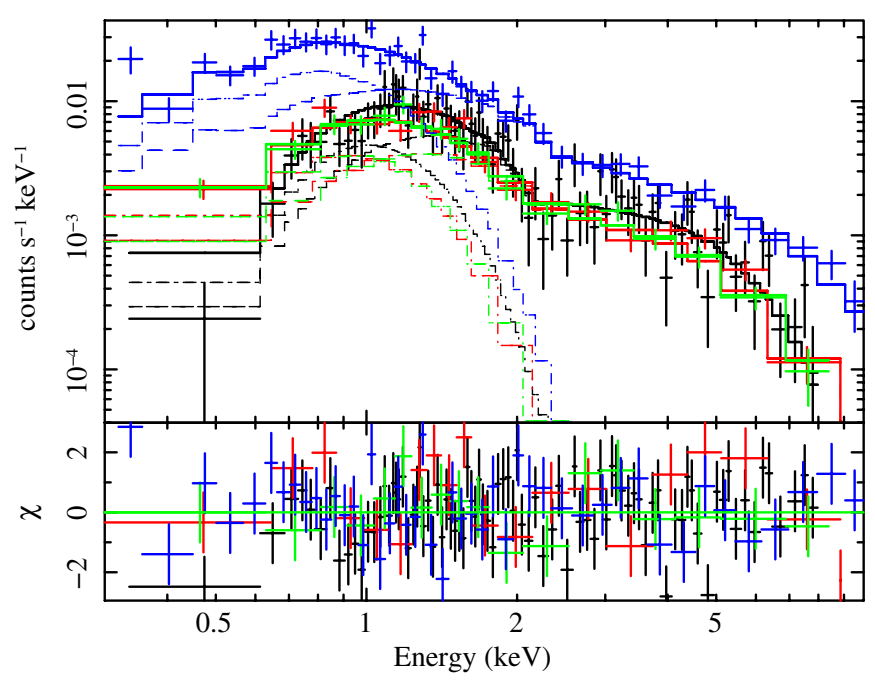

Figure 9. ACIS-I (black), EPIC MOS1 (red), MOS2 (green), and PN (blue) spectra of the pulsar region (20" radius) fitted with the absorbed PL (dashed line) + BB (dash-dotted line) model.

(A color version of this figure is available in the online journal.)

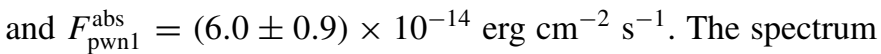
of PWN2 was also binned with a minimum of 10 counts per bin and fitted with the absorbed PL model in the same energy range. We used the $C$-statistic because of small number of counts in the region. The PL fit to the spectrum of the PWN2 gives somewhat larger $\Gamma=1.8_{-0.9}^{+1.0}$; however, the uncertainties are large as one can see from the confidence contours shown in Figure 10. We also extracted and fitted the spectrum from both regions combined, since the difference in slopes of both spectra is not statistically significant. The fit gives $n_{\mathrm{H}, 21}=3.7_{-1.3}^{+2.0}, \Gamma=1.37 \pm 0.22$, and the absorbed flux is $F_{\text {pwn1+2 }}^{\mathrm{abs}}=(7 \pm 1) \times 10^{-14} \mathrm{erg} \mathrm{cm}^{-2} \mathrm{~s}^{-1}$ in the $0.3-8 \mathrm{keV}$ band, while the unabsorbed flux is $F_{\text {pwn } 1+2}^{\text {unabs }}=$ $9_{-1}^{+2} \times 10^{-14} \mathrm{erg} \mathrm{cm}^{-2} \mathrm{~s}^{-1}$ in the same energy band. It corresponds to the luminosity $L_{\mathrm{PWN} 1+2} \sim 7 \times 10^{31} d_{2.5}^{2} \mathrm{erg} \mathrm{s}^{-1}$, i.e., about $130 \%$ of the pulsar's non-thermal luminosity estimated from the same observation.

For the PWN region on the I1 chip (see Figure 1), we binned the spectrum with minimum 25 counts per bin and fitted with the absorbed PL model in the $0.3-8 \mathrm{keV}$ energy range. We fixed the hydrogen column density to the value $n_{\mathrm{H}, 21}=4.7$, found from the PL+BB model for pulsar obtained from the

Table 2

Absorbed BB+PL Fits to the Pulsar (or Pulsar + Compact PWN) Spectrum from the Chandra and XMM-Newton Observations

\begin{tabular}{|c|c|c|c|c|c|c|c|c|}
\hline Model $^{\mathrm{a}}$ & $n_{\mathrm{H}, 21}$ & $\Gamma$ & $\mathcal{N}^{\mathrm{b}}$ & $k T^{\mathrm{c}}$ & $\mathcal{A}^{\mathrm{d}}$ & $F_{\mathrm{abs}}{ }^{\mathrm{e}}$ & $F_{\text {unabs }}^{\mathrm{f}}$ & $\left(C\right.$ or $\left.\chi_{v}^{2}\right) /$ dof $^{g}$ \\
\hline Chandra $\left(1^{\prime \prime}\right)$ & $4.7_{-2.8}^{+3.6}$ & $1.72_{-0.63}^{+0.55}$ & $9.8_{-5.8}^{+9.1}$ & $0.14_{-0.04}^{+0.06}$ & $13_{-12}^{+454}$ & $6.7_{-0.8}^{+0.9}$ & $32_{-21}^{+190}$ & $28.2 / 29$ \\
\hline XMM-Newton $\left(20^{\prime \prime}\right)$ & $2.2_{-0.7}^{+1.0}$ & $1.35_{-0.17}^{+0.16}$ & $22 \pm 5$ & $0.18_{-0.03}^{+0.04}$ & $2_{-1}^{+6}$ & $17.8 \pm 0.8$ & $26_{-4}^{+8}$ & $1.27 / 80$ \\
\hline Chandra $\left(20^{\prime \prime}\right)$ & $2.4_{-1.9}^{+3.1}$ & $1.32_{-0.69}^{+0.50}$ & $18_{-12}^{+18}$ & $0.22_{-0.09}^{+0.13}$ & $0.5_{-0.4}^{+22}$ & $16 \pm 2$ & $22_{-5}^{+31}$ & $1.03 / 82$ \\
\hline Combined $\left(20^{\prime \prime}\right)$ & $1.9_{-0.6}^{+0.8}$ & $1.33_{-0.17}^{+0.16}$ & $21 \pm 5$ & $0.19_{-0.03}^{+0.04}$ & $0.9_{-0.6}^{+2.8}$ & $17.3 \pm 0.7$ & $24_{-3}^{+5}$ & $1.18 / 167$ \\
\hline
\end{tabular}

Notes. The errors shown represent $90 \%$ confidence intervals.

${ }^{a}$ Radius of extraction region is given in parentheses.

b Spectral flux in units of $10^{-6}$ photons $\mathrm{cm}^{-2} \mathrm{~s}^{-1} \mathrm{keV}^{-1}$ at $1 \mathrm{keV}$.

c $\mathrm{BB}$ temperature in $\mathrm{keV}$.

${ }^{\mathrm{d}}$ Projected emitting area for BB model in units of $\mathrm{km}^{2}$ at the distance of $2.5 \mathrm{kpc}$.

e Absorbed flux in the $0.3-8 \mathrm{keV}$, in units of $10^{-14} \mathrm{erg} \mathrm{cm}^{-2} \mathrm{~s}^{-1}$.

${ }^{\mathrm{f}}$ Unabsorbed flux in the $0.3-8 \mathrm{keV}$, in units of $10^{-14} \mathrm{erg} \mathrm{cm}^{-2} \mathrm{~s}^{-1}$.

g Best-fit $C$-statistic value for Chandra observation ( $1^{\prime \prime}$ radius aperture) or reduced $\chi^{2}$ value (other cases). 


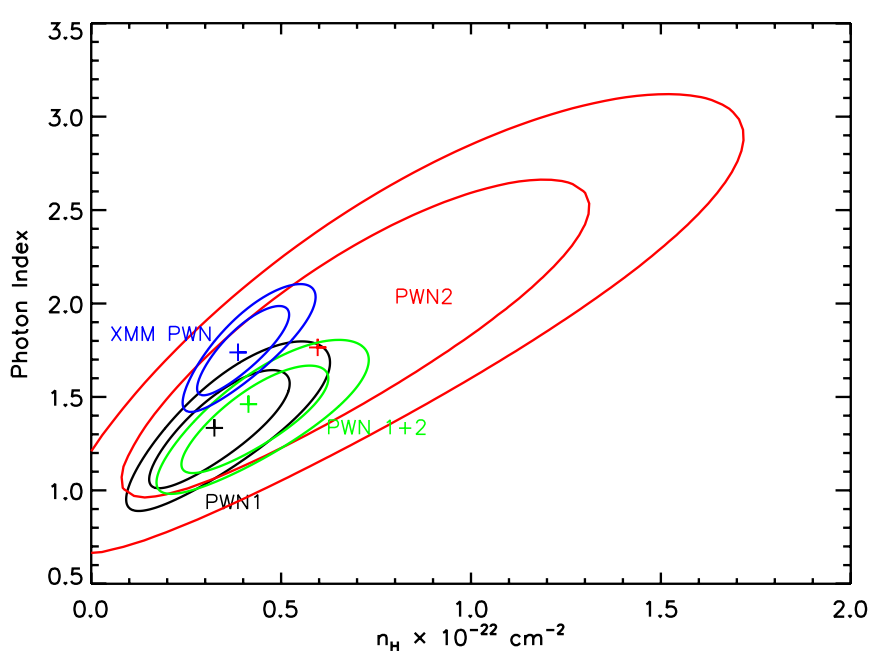

Figure 10. Confidence contours $(68 \%$ and $90 \%)$ in the $n_{\mathrm{H}}-\Gamma$ plane for the PWN spectrum measured with XMM-Newton (blue) and the PWN1 (black), PWN2 (red), and PWN 1+2 (green) spectra measured with Chandra.

(A color version of this figure is available in the online journal.)

Chandra data (see Table 2). The fit $\left(\chi_{v}^{2}=0.66\right.$ for 10 dof) gives $\Gamma=2.5_{-0.9}^{+1.8}$ and $\mathcal{N}_{\Gamma}=8_{-4}^{+6} \times 10^{-6}$ photons $\mathrm{cm}^{-2} \mathrm{~s}^{-1} \mathrm{keV}^{-1}$ at $1 \mathrm{keV}$. The absorbed flux in the same energy range is $1.3_{-0.7}^{+0.9} \times 10^{-14} \mathrm{erg} \mathrm{cm}^{-2} \mathrm{~s}^{-1}$, while the unabsorbed flux is $4_{-1}^{+10} \times 10^{-14} \mathrm{erg} \mathrm{cm}^{-2} \mathrm{~s}^{-1}$. The spectrum of the PWN region on the $\mathrm{I} 2$ chip was binned with a minimum of 50 counts per bin and fitted with a single absorbed PL model in the energy of $0.3-8 \mathrm{keV}$. We fixed the hydrogen column density of $n_{\mathrm{H}, 21}=$ 4.7. The fit was acceptable $\left(\chi_{v}^{2}=0.7\right.$ for 18 dof $)$ with $\Gamma=$ $1.7 \pm 0.4$ and $\mathcal{N}_{\Gamma}=(1.5 \pm 0.5) \times 10^{-5}$ photons $\mathrm{cm}^{-2} \mathrm{~s}^{-1} \mathrm{keV}^{-1}$ at $1 \mathrm{keV}$. The absorbed flux in the same energy range is $(7 \pm 3) \times 10^{-14} \mathrm{erg} \mathrm{cm}^{-2} \mathrm{~s}^{-1}$, while the unabsorbed flux is $(9 \pm 2) \times 10^{-14} \mathrm{erg} \mathrm{cm}^{-2} \mathrm{~s}^{-1}$.

Figure 11 shows the MOS1 and MOS2 spectra of the region shown in Figure 3, binned with minimum 50 counts per bin. The region is much larger than that of PWN1+PWN2 of Chandra (see Figures 1 and 3). The absorbed PL fit is good $\left(\chi_{v}^{2}=0.94\right.$ for 61 dof). The best-fit hydrogen column density and photon index are $n_{\mathrm{H}, 21}=3.9_{-1.1}^{+1.5}$ and $\Gamma=1.7_{-0.2}^{+0.3}$, respectively. The absorbed flux is $F_{\mathrm{pwn}}^{\mathrm{abs}}=(5.0 \pm 0.6) \times 10^{-13} \mathrm{erg} \mathrm{cm}^{-2} \mathrm{~s}^{-1}$ in $0.3-8 \mathrm{keV}$. The unabsorbed flux $F_{\mathrm{pwn}}^{\mathrm{unabs}}=7.5_{-0.9}^{+1.4} \times 10^{-13} \mathrm{erg} \mathrm{cm}^{-2} \mathrm{~s}^{-1}$. The results of the fits, summarized in Table 3, suggest that the PWN spectrum softens with the distance from the pulsar. The combined compact+extended PWN flux is $F_{\mathrm{pwn}}^{\text {unabs }} \approx 9 \times$

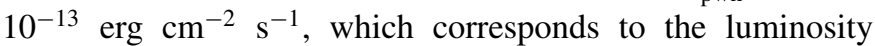
$L_{\mathrm{pwn}}=6.7 \times 10^{32} d_{2.5}^{2} \mathrm{erg} \mathrm{s}^{-1} \approx 2.8 L_{\mathrm{psr}}$.

\subsubsection{Timing Analysis}

We searched for pulsations in the PN data (time resolution of $6 \mathrm{~ms}$ ) extracted from the $r=20^{\prime \prime}$ circle centered at the pulsar position, which contains 939 counts, including $15 \%$ from the background, in the $0.3-1.1 \mathrm{keV}$ band, where the $\mathrm{BB}$ component is dominant (see Figure 9). From the same region in the 1.1-10 keV band, we extracted 1473 counts, including $21 \%$ from the background. The photon arrival times were corrected to the solar system barycenter using the SAS barycen tool. The PN observation started at the epoch 55,037.63605 MJD and continued for the time $T_{\text {span }}=55.5 \mathrm{ks}$. We used the radio timing ephemeris reported by Camilo et al. (2004) $(f=$ $6.0201677726(4) \mathrm{s}^{-1}$ and $\dot{f}=-1.305395(4) \times 10^{-11} \mathrm{~s}^{-2}$

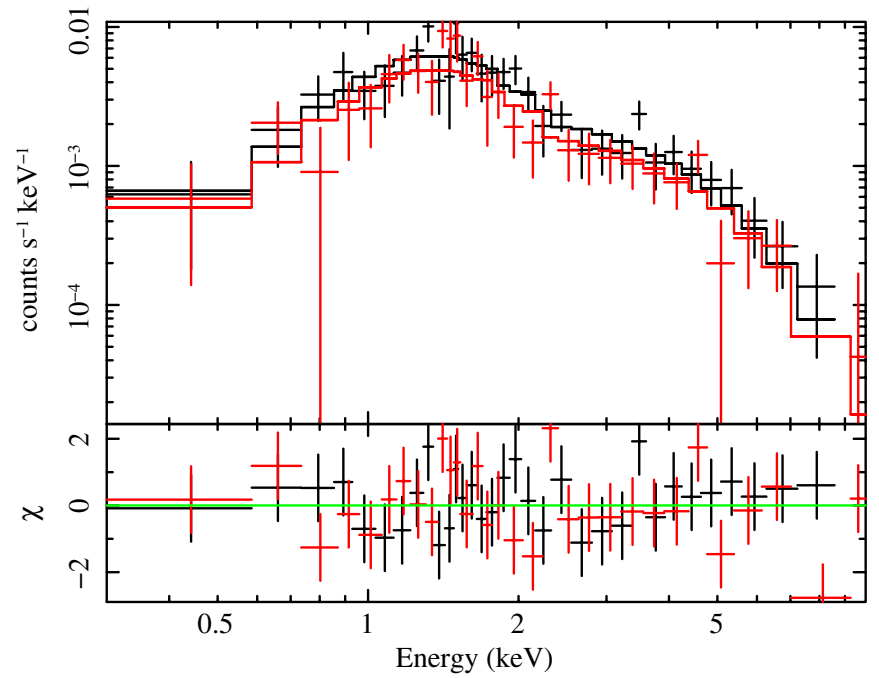

Figure 11. EPIC MOS1 (black) and MOS2 (red) spectra of the PWN shown in Figure 3.

(A color version of this figure is available in the online journal.)

for the epoch of 52,921.0 MJD) to calculate the predicted frequency at our epoch. There was a glitch at the epoch of $52,021 \pm 16 \mathrm{MJD}$ ( $1 \sigma$ uncertainties), but it was before the ephemeris was measured. We assumed that there were no glitches after that. The predicted frequency for our observation is $f_{\text {predict }}=6.017780858(7) \mathrm{Hz}$.

We applied the $Z_{1}^{2}$ test (Buccheri et al. 1983; Pavlov et al. 1999) to search for the pulsed signal in the band $6.017780 \pm$ $0.000010 \mathrm{~Hz}$ near the predicted frequency, with a step of $0.1 \mu \mathrm{Hz} \approx 0.006 T_{\text {span }}^{-1}$. We found the maximum $Z_{1, \max }^{2}=40.5$ at $f=6.0177801 \mathrm{~Hz} \pm 1.6 \mu \mathrm{Hz}$, i.e., the pulsations are detected with a $6 \sigma$ significance in the $0.3-1.1 \mathrm{keV}$ energy range. ${ }^{14}$ The maximum value of $Z_{1}^{2}$ in the $1.1-10 \mathrm{keV}$ energy range is only 7.9 at $f=6.0177819 \mathrm{~Hz} \pm 3.5 \mu \mathrm{Hz}$, which corresponds to $2.3 \sigma$ significance.

Using the two epochs $(55,037.63605$ MJD from our observation and 52,921.0 MJD from the radio) and two frequency values, we can estimate the frequency derivative, $\dot{f}=$ $-1.3056(7) \times 10^{-11} \mathrm{~s}^{-2}$, which is consistent with $\dot{f}$ determined from the radio. Figure 12 shows the pulse profiles with 10 phase bins, with the estimated pulsed fraction (the ratio of the number of counts above the minimum level to the total number of counts) $p=36 \% \pm 5 \%$ in $0.3-1.1 \mathrm{keV}, 18 \% \pm 4 \%$ in $1.1-10 \mathrm{keV}$, and $14 \% \pm 3 \%$ in $0.3-10 \mathrm{keV}$. We estimated the error of pulsed fraction as $\delta p=\sqrt{2 / N}$ (Linsley 1975). After correcting for the background, we obtain the intrinsic pulsed fraction $p_{\text {int }}=42 \% \pm 5 \%, 23 \% \pm 4 \%$, and $17 \% \pm 3 \%$, respectively. Thus, we conclude that the X-ray emission from J1357 is undoubtedly pulsed, although the pulsed fraction is not as high as reported by Zavlin (2007). Interestingly, the pulsed fraction is higher at lower photon energies, where the thermal component dominates. Such strong pulsations of the thermal emission are likely due to the anisotropy of atmospheric radiation in the strong magnetic field of this pulsar.

\footnotetext{
${ }^{14}$ The $1 \sigma$ frequency uncertainty is calculated as $\delta f=3^{1 / 2} \pi^{-1} T_{\mathrm{span}}^{-1}\left(Z_{1, \max }^{2}\right)^{-1 / 2}$. This equation can be derived using the method outlined in Section 2.4 of Bretthorst (1988). The numerical factor, $\sqrt{3} / \pi \approx 0.55$, was confirmed in Monte Carlo simulations by A. Kaurov (2011, private communication).
} 
Table 3

Spectral Properties of the PWN from the Chandra and XMM-Newton Observations

\begin{tabular}{lcccccc}
\hline \hline Region & $n_{\mathrm{H}, 21}$ & $\Gamma$ & $\mathcal{N}^{\mathrm{a}}$ & $F_{\text {abs }}{ }^{\mathrm{b}}$ & $F_{\text {unabs }}{ }^{\mathrm{c}}$ & $\left(C\right.$ or $\left._{\nu}^{2}\right) /$ dof $^{\mathrm{d}}$ \\
\hline Chandra & & & & & & \\
PWN1 & $3.2_{-1.4}^{+2.3}$ & $1.31_{-0.33}^{+0.37}$ & $8.5_{-2.9}^{+4.7}$ & $6.0 \pm 0.9$ & $7 \pm 1$ & $1.3 / 24$ \\
PWN2 & $6.0_{-5.5}^{+7.9}$ & $1.8_{-0.9}^{+1.0}$ & $3.7_{-3.7}^{+9.5}$ & $1.3_{-0.4}^{+0.5}$ & $2.2_{-0.8}^{+4.2}$ & $1.04 / 5$ \\
PWN 1+2 & $3.7_{-1.3}^{+2.0}$ & $1.37 \pm 0.22$ & $12_{-4}^{+6}$ & $7 \pm 1$ & $9_{-1}^{+2}$ & $0.90 / 31$ \\
PWN I1 & 4.7 (fixed) & $2.5_{-0.9}^{+1.8}$ & $8_{-4}^{+6}$ & $1.3_{-0.7}^{+0.9}$ & $4_{-1}^{+10}$ & $0.66 / 10$ \\
PWN I2 & 4.7 (fixed) & $1.7 \pm 0.4$ & $15 \pm 5$ & $7 \pm 3$ & $9 \pm 2$ & $0.70 / 18$ \\
XMM-Newton & & & & & & \\
PWN & $3.9_{-1.1}^{+1.5}$ & $1.74_{-0.24}^{+0.27}$ & $123_{-31}^{+46}$ & $50 \pm 6$ & $75_{-9}^{+14}$ & $0.94 / 61$ \\
\hline
\end{tabular}

Notes. The errors shown represent $90 \%$ confidence intervals.

${ }^{\text {a }}$ Spectral flux in units of $10^{-6}$ photons $\mathrm{cm}^{-2} \mathrm{~s}^{-1} \mathrm{keV}^{-1}$ at $1 \mathrm{keV}$.

${ }^{\mathrm{b}}$ Absorbed flux in the $0.3-8 \mathrm{keV}$, in units of $10^{-14} \mathrm{erg} \mathrm{cm}^{-2} \mathrm{~s}^{-1}$.

${ }^{\mathrm{c}}$ Unabsorbed flux in the $0.3-8 \mathrm{keV}$, in units of $10^{-14} \mathrm{erg} \mathrm{cm}^{-2} \mathrm{~s}^{-1}$.

${ }^{\mathrm{d}} C$-statistic value for PWN2, $\chi^{2}$ values for other regions.

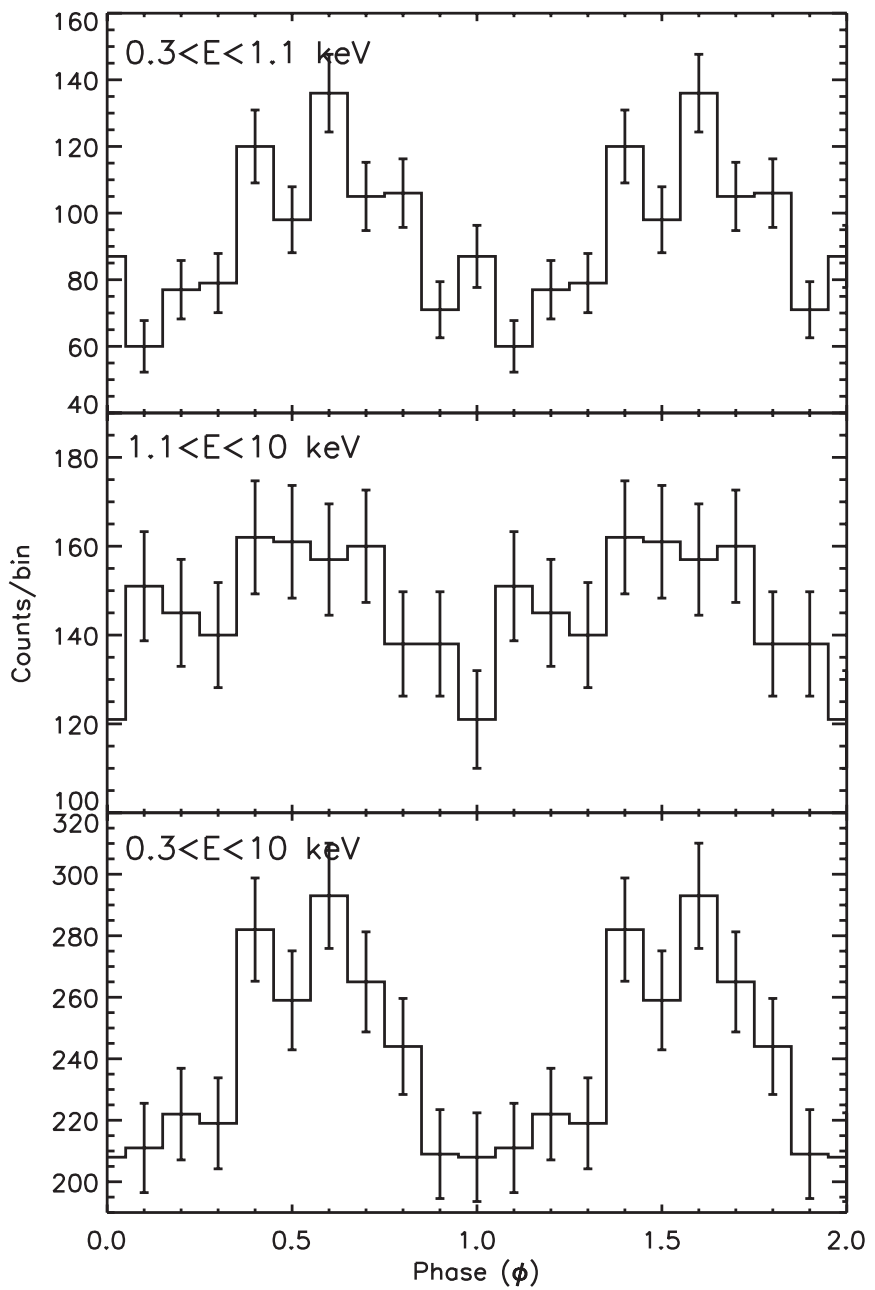

Figure 12. Pulse profiles of J1357 extracted from the EPIC PN data in three energy bands.

\section{DISCUSSION}

Our Chandra ACIS and XMM-Newton EPIC observations have shown that the PWN of J1357 is much more extended and luminous than could be seen from the earlier observations. We have also found that the thermal component of pulsar emission is strongly pulsed, while the pulsation of the non- thermal component, which dominates at higher energies, is much weaker. Below we discuss the physical implications of our findings, compare the J1357 PSR/PWN properties to those of other similar pulsars observed in X-rays, and investigate the relation between J1357, HESS J1356, and putative host SNR G309.8-2.6.

\subsection{Pulsar}

The pulsar spectrum cannot be satisfactory fitted by simple one-component models, such as absorbed PL or BB (see Section 2.2.1). The two-component BB+PL model fits the spectrum much better, although some residuals are still present in the combined fits. The best-fit photon index for the PL component, $\Gamma \simeq 1.7 \pm 0.6$, is marginally larger than $\Gamma \simeq$ $1.3 \pm 0.3$ of the compact PWN (region PWN1). The nonthermal luminosity is $L_{\text {nonth }} \approx 5 \times 10^{31} \mathrm{erg} \mathrm{s}^{-1}$ in the energy range $0.3-8 \mathrm{keV}$. The PL component may partly come from an unresolved PWN. This could explain the weak pulsations above $1 \mathrm{keV}$ (see Section 2.2.3), in contrast to, for instance, the Vela pulsar (which is well resolved from the surrounding PWN), whose pulsed fraction grows with energy reaching $62 \%$ above $1.8 \mathrm{keV}$ (Sanwal et al. 2002). Our timing analysis limits the luminosity of the unresolved PWN to $\lesssim 77 \%$ of the point-source luminosity in $1.1-10 \mathrm{keV}$.

Unlike the hard non-thermal component, the soft thermal component should not be strongly contaminated by the PWN emission since it clearly dominates at $\lesssim 1 \mathrm{keV}$. Therefore, the best-fit $\mathrm{BB}$ temperature, $T \approx 1.9-2.7 \mathrm{MK}$, radius, $R \sim$ $0.3-1.1 \mathrm{~km}$, and bolometric luminosity, $L_{\mathrm{bol}} \sim 2 \times 10^{31} \mathrm{erg} \mathrm{s}^{-1}$, are reasonably accurately determined (see Table 2 ). The values are similar to those of other Vela-like pulsars. Although the temperature is a factor of two higher than expected from the standard NS cooling, and the emitting area radius is a factor of 10 smaller than the classical NS radius, the discrepancies can be attributed to the use of the simplistic BB model. Indeed, the fit with the hydrogen atmosphere models (e.g., Pavlov et al. 1995), described in Section 2.2.1, gives a lower temperature, $T_{\text {eff }}^{\infty}=0.70-0.78 \mathrm{MK}$ at $R^{\infty}=13 \mathrm{~km}$, at the same bolometric luminosity. The measured bolometric luminosity is a factor of $\sim 3$ lower than predicted by the theoretical cooling curve for low-mass NSs $\left(M_{\mathrm{NS}}=1.3 M_{\odot}\right)$ without superfluidity; it is consistent with the cooling curves for heavier NSs (e.g., $M_{\mathrm{NS}}=1.5-1.6 M_{\odot}$ ) for some superfluidity models (Yakovlev $\&$ Pethick 2004). 
Very surprising is the high $(\approx 40 \%)$ pulsed fraction associated with the thermal component. In general, one should not expect such strong pulsations from a nonuniformly heated NS surface with dipolar magnetic field for a BB-like angular distribution. However, there have been recent reports of even stronger thermal pulsations in strongly magnetized young pulsars, such as PSR J1119-6127 (74\% $\pm 14 \%$; Gonzalez et al. 2005) and PSR J1718-3718 (52\% $\pm 13 \%$; Zhu et al. 2011). This suggests that the strong pulsations are caused by the combined effect of the anisotropic temperature distribution and strong anisotropy of emitted radiation in high magnetic fields (Pavlov et al. 1994). An alternative possibility is that part of emission interpreted as thermal from our $\mathrm{BB}+\mathrm{PL}$ and $\mathrm{NSA}+\mathrm{PL}$ fits is in fact an additional, softer non-thermal component, which is masked at higher energies by the harder, unresolved PWN emission. This unaccounted component might be the reason for the remaining residuals of the PL+BB and NSA+PL fits. However, higher quality data are needed to test this hypothesis.

\section{2. $P W N$}

At the plausible distance of $2.5 \mathrm{kpc}$, the unabsorbed X-ray luminosity of the compact $\mathrm{PWN}, L_{\mathrm{pwn} 1} \approx 5 \times 10^{31} \mathrm{erg} \mathrm{s}^{-1}$ in the $0.3-8 \mathrm{keV}$ band, corresponds to the X-ray efficiency, $\eta_{\text {pwn } 1} \equiv L_{\text {pwn1 }} / \dot{E} \sim 1.7 \times 10^{-5}$, lower than those of most Velalike pulsars (see Kargaltsev et al. 2007; KP08). This suggests that either the distance is underestimated or, in addition to $\dot{E}$ and $\tau, \eta_{\text {pwn } 1}$ strongly depends on other factors (e.g., the pulsar's speed and the angle between the spin and magnetic axes). Note, however, that the efficiency of the entire detected PWN is higher by a factor of $15, \eta_{\text {pwn }} \sim 2.6 \times 10^{-4}$.

The spectral slope of the compact PWN, $\Gamma_{\text {pwn1 }}=1.3 \pm 0.3$, is similar to those of PWNe around Vela-like pulsars (listed in Table 2 of Kargaltsev et al. 2007). The PWN spectrum apparently softens with the distance from the pulsar, becoming $\Gamma \approx 1.7-1.8$ for the narrow feature (PWN2) and the largescale PWN, which suggests synchrotron cooling of the outflow. The degree of spectral softening is similar to that seen for the extended tail of PSR J1509-5850, where the photon index changes from $\Gamma=1.8 \pm 0.3$ for PWN in the vicinity of the pulsar to $2.4 \pm 0.4$ for the extended tail (Kargaltsev et al. 2008).

Our X-ray images reveal the morphology of the PWN on different angular scales. The observed morphology does not easily fit in either the bow-shock or torus-jet category (see KP08). The bright compact PWN1 extends up to $\simeq 10^{\prime \prime}$ from the pulsar and has rather amorphous, somewhat asymmetric morphology. The diffuse emission is noticeably brighter northeast of the pulsar compared to the opposite side, although some emission is still clearly discernible within the PWN1 region southwest of the pulsar. We find no evidence of structures that could be interpreted as a torus associated with the TS in the pulsar wind, which may suggest that the TS occurs at an angular distance of several arcseconds or less, corresponding to $r_{s} \lesssim 4 \times 10^{16} d_{2.5} \mathrm{~cm}$ (versus $1 \times 10^{17} \mathrm{~cm}$ in the Vela PWN; Helfand et al. 2001). If the pulsar is moving subsonically inside the hot SNR medium, we can use the above estimate to obtain a lower limit on the ambient pressure at $p_{\mathrm{amb}} \sim \dot{E}\left(4 \pi c r_{s}^{2}\right)^{-1} \gtrsim 5 \times 10^{-9}\left(r_{s} / 4 \times 10^{16} \mathrm{~cm}\right)^{-2} \mathrm{dyn} \mathrm{cm}^{-2}$, which is close to the maximum pressure one can expect in a 10 kyr old SNR (Kargaltsev et al. 2009; Bamba et al. 2010). The high pressure required, and the asymmetry of the compact PWN, elongated approximately along the northeast-southwest direction, might be indicative of the TS also being asymmetric, i.e., being farther away from the pulsar on the northeast side than on the southwest side. Such a situation may occur if the pulsar is moving fast through the surrounding medium in the southwest direction, and the ram pressure of the medium compresses the PWN in front of the pulsar. If the pulsar's speed exceeds the sound speed in the medium, $c_{s}=\left(5 k T / 3 \mu \mathrm{m}_{\mathrm{H}}\right)^{1 / 2}=12 \mu^{-1 / 2} T_{4}^{1 / 2} \mathrm{~km} \mathrm{~s}^{-1}$, a bow shock is formed. The appearance of a bow shock PWN depends on the Mach number, uniformity of the medium, and intrinsic anisotropy of the pulsar wind. X-ray PWNe around supersonically moving pulsars often exhibit a bright "bullet" in the vicinity of the pulsar and a much more extended faint tail in the direction opposite to that of the pulsar motion; however, more complex structures have been seen in some cases (e.g., the Guitar and Eel PWNe associated with B2224+65 and J1826-1256, respectively; Johnson \& Wang 2010; Roberts et al. 2007).

The adjacent PWN2 region shown in Figure 1 encompasses the fainter narrow feature attached to the compact PWN. Interestingly, this narrow feature bends sharply at about $23^{\prime \prime}$ from the pulsar. Although there is no doubt that the feature is part of the PWN, the origin of the feature is not clear. For instance, it could be a pulsar jet. Indeed, some pulsar jets are known to show extreme bending (e.g., the outer jet of the Vela pulsar; Pavlov et al. 2003). The apparent lack of a similarly looking counterjet should not be surprising since the two jets can differ significantly both in shape and surface brightness due to the Doppler boost and/or proper motion effects (e.g., Pavlov et al. 2003). On the other hand, the ratio of the compact PWN luminosity to that of the putative jet, $L_{\mathrm{pwn} 1} / L_{\mathrm{pwn} 2} \sim 5$, is noticeably larger than that in the Vela and Crab PWNe but comparable to that of the PWN around PSR B1706-44 (Romani et al. 2005). If this interpretation is taken at face value, one could expect the pulsar to be moving northeast (in general, jets tend to be co-aligned with the pulsar's direction of motion-cf. the Vela pulsar; Pavlov et al. 2003).

Alternatively, the narrow feature could be a part (e.g., an inner channel) of the pulsar tail (see, e.g., Kargaltsev \& Pavlov 2008 and references therein). In this case the pulsar would be moving in the opposite (southwest) direction. This interpretation is in line with the above-described asymmetry of the compact PWN whose brighter part could, in this case, indeed be the "bullet" associated with the TS deformed by the ram pressure of the oncoming ISM. However, the sharp bending and the lack of a fainter, much more extended tail (cf. J1509-5850 tail; Kargaltsev \& Pavlov 2008), and the emission west-southwest of the compact PWN, are at odds with this interpretation. ${ }^{15}$

Finally, the narrow feature could be a part of the bow associated with the forward shock (see, e.g., the Geminga PWN; Pavlov et al. 2006, 2010) with the pulsar moving southeast. In this case the observed large-scale emission can also be associated with the asymmetric bow shock similar to that seen around PSR J1826-1256 (Roberts et al. 2007). Somewhat puzzling may be the lack of any X-ray emission within the bow interior; however, this is also the case in the Guitar and Eel bow shocks. To conclude, with the data in hand we cannot univocally interpret the morphology of the X-ray PWN. Measuring the pulsar's proper motion should provide decisive information allowing one to discriminate between the above interpretations. Assuming a typical pulsar speed of $400 \mathrm{~km} \mathrm{~s}^{-1}$ (Hobbs et al. 2005), this should be possible to accomplish in several years with very long baseline interferometry.

\footnotetext{
15 The diffuse emission seen in the PMN 4.85 GHz image north of the pulsar (panel (e) in Figure 6) could, in principle, be the radio counterpart of the extended pulsar tail. Deeper radio observations are required to test this hypothesis.
} 
Table 4

Properties of Candidate TeV Plerions and Their Parent Pulsars

\begin{tabular}{|c|c|c|c|c|c|c|c|c|c|c|}
\hline HESS ID & $f_{\gamma}{ }^{\mathrm{a}}$ & $\begin{array}{c}\text { TeV Size } \\
\text { (pc) }\end{array}$ & Offset $^{b}$ & PSR & $\dot{E}_{36}{ }^{\mathrm{c}}$ & $\begin{array}{c}\tau \\
(\mathrm{kyr})\end{array}$ & $\begin{array}{l}\text { Dist. } \\
\text { (kpc) }\end{array}$ & $L_{\gamma} / \dot{E}$ & $L_{X} / L_{\gamma}$ & $\mathcal{B}_{\text {acis }}{ }^{\mathrm{d}}$ \\
\hline J1809-193 & 0.3 & 20 & $8^{\prime}$ & J1809-1917 & 1.8 & 50 & 3.5 & $1 \%$ & $2 \%$ & 8 \\
\hline Vela X & 0.75 & 5 & $30^{\prime}$ & $\mathrm{J} 0835-4510$ & 6.9 & 11 & 0.3 & $0.002 \%$ & $90 \%$ & $\ldots$ \\
\hline $\mathrm{J} 1825-137$ & 0.48 & 70 & $10^{\prime}$ & J1826-1334 & 2.8 & 21 & 3.5 & $4 \%$ & $0.3 \%$ & 7.5 \\
\hline $\mathrm{J} 1356-645$ & 0.79 & 40 & $8^{\prime}$ & $\mathrm{J} 1357-6429$ & 3.1 & 7.3 & 2.5 & $0.2 \%$ & $10 \%$ & 19 \\
\hline
\end{tabular}

Notes.

${ }^{a}$ Unabsorbed $\gamma$-ray flux (1-10 TeV) in units of $10^{-11} \mathrm{erg} \mathrm{s}^{-1} \mathrm{~cm}^{-2}$.

${ }^{\mathrm{b}}$ Offset of the TeV source center from the pulsar.

c Pulsar spin-down power, $\dot{E}$, in units of $10^{36} \mathrm{erg} \mathrm{s}^{-1}$

d Average surface brightness measured from Chandra ACIS images, in counts ks ${ }^{-1} \operatorname{arcmin}^{-2}$.

\subsection{The Nature of the VHE Source}

Given the lack of other promising counterparts and the location of J1357 within the HESS J1356 extent, it seems very plausible that the two should be related in some way. Yet the nature of HESS J1356 has not been firmly established. The TeV emission could be attributed to either the host SNR or to the relic PWN of J1357. To date, several well-known SNRs have been detected in the TeV band (e.g., Aharonian et al. 2004). In the cases of resolved SNRs, the TeV emission was associated with the SNR shell, and the sizes of the SNRs in TeV images were similar to those of the non-thermal radio shells, indicating that both emission components are powered by particles accelerated in the forward shock. Although no shell is seen in the radio images of HESS J1356, the radio emission is mainly seen near the center of HESS J1356 (see Figure 6). Thus, in this case the $\mathrm{TeV}$ emission does not appear to be associated with the non-thermal shell, and the shell itself is not seen in the radio.

An alternative, more plausible, interpretation of the $\mathrm{TeV}$ and radio emission could be a relic PWN. Many extended $\mathrm{TeV}$ sources neighbor young Vela-like pulsars, sometimes offset up to $10^{\prime}-20^{\prime}$ from the center of $\mathrm{TeV}$ emission (see KP10 for recent review). Furthermore, recent X-ray observations of the Vela pulsar region (Vela X; Mori et al. 2008), PSR J1826-1334 (Gaensler et al. 2003; Pavlov et al. 2008), and PSR J1809-1917 (Kargaltsev \& Pavlov 2007) have provided convincing evidence that the TeV sources are connected to the parent pulsars through faint asymmetric X-ray nebulae ${ }^{16}$ (see Table 4). Indeed, the Chandra observations have demonstrated that in the above three examples the PWNe consist of compact (0.1-0.8 pc) bright cores and fainter asymmetric, more extended (2-8 pc) components. The offsets and the asymmetries of the X-ray PWNe could be created by the reverse SNR shock that had propagated through the nonhomogeneous SNR interior and reached one side of the PWN sooner than the other side (Blondin et al. 2001). This scenario could also account for the similarly asymmetric, offset TeV emission (e.g., de Jager \& Djannati-Ataï 2009). However, the physical origin of the $\mathrm{TeV}$ emission still remains under debate. It can be produced via the inverse Compton scattering of the relativistic pulsar wind electrons on photons from the omnipresent cosmic microwave background, galactic IR background, and IR photons from local star-forming regions and warm dust clouds. Alternatively, the $\mathrm{TeV}$ photons can be produced as a result of $\pi^{0} \rightarrow \gamma+\gamma$ decay, with $\pi^{0}$ being produced when the relativistic protons from the pulsar wind interact with the ambient matter (e.g., Horns et al. 2007). In the

\footnotetext{
16 A large fraction of relic PWN candidates remains where only a bright compact PWN is seen in X-rays while the extended component is not seen perhaps due to is faintness and/or rapid cooling of the outflow.
}

case of HESS J1356, the offset is well within the range observed in other relic PWN candidates (see KP10). The ratio of the TeV luminosity to the pulsar's spin-down power, $L_{\mathrm{TeV}} / \dot{E} \sim 0.002$, and the ratio of X-ray to $\gamma$-ray $(1-10 \mathrm{TeV}) \mathrm{PWN}$ luminosities, $L_{X} / L_{\mathrm{TeV}} \sim 0.1$, are similar to those of other relic plerions.

Alternatively, the relic PWN could be left behind the fast moving pulsar if the latter is moving in the northeast direction (see above). At a typical speed of $400 \mathrm{~km} \mathrm{~s}^{-1}$, the pulsar would move by 5.6 in $10 \mathrm{kyr}$, which is consistent with the offset between the pulsar and the center of HESS J1356. So far, there have been no reports of very high energy (VHE) emission from pulsar tails. It would be interesting to establish the presence of the $\mathrm{TeV}$ emission from an extended pulsar tail because this would help to break the degeneracy in interpreting the nature of the VHE emission from the crushed PWNe, for which both leptonic (inverse Compton) and hadronic ( $\pi^{0}$ decay) $\mathrm{TeV}$ emission mechanisms are currently being debated. If the $\pi^{0}$ decay is the dominant process in the crushed PWNe, we would expect pulsar tails to be significantly fainter in the $\mathrm{TeV}$ because high-speed pulsars move in low-density media outside their host SNRs. On the other hand, in the leptonic scenario, crushed plerions and pulsar tails should have comparable TeV luminosities. Regardless of the nature of the relic PWN and the mechanism responsible for the VHE emission, one can expect that the observed extended radio emission within HESS J1356 may also be produced by the pulsar wind if the electron spectral energy distribution extends to sufficiently low energies. Better quality radio images, more complete multiwavelength spectrum, and robust multizone pulsar wind models are required to assess the origin of the radio emission.

The work on this project was partly supported by Chandra award GO0-11070X, NASA grants NNX09AT11G, NNX09AC81G, and NNX09AC84G, and National Science Foundation grants AST09-08733 and AST09-08611. The work by G.G.P. and Y.A.S. was partly supported by the Ministry of Education and Science of the Russian Federation (contract 11.G34.31.0001). We thank Alexander Kaurov for his help with simulations of timing uncertainties.

\section{REFERENCES}

Abramowski, A., Acero, F., Aharonian, F., et al. 2011, A\&A, 533, A103

Aharonian, F. A., Akhperjanian, A. G., Aye, K.-M., et al. 2004, Nature, 432, 75

Bamba, A., Mori, K., \& Shibata, S. 2010, ApJ, 709, 507

Blondin, J. M., Chevalier, R. A., \& Frierson, D. M. 2001, ApJ, 563, 806

Bretthorst, G. L. 1988, Bayesian Spectrum Analysis and Parameter Estimation (New York: Springer)

Buccheri, R., Bennett, K., Bignami, G. F., et al. 1983, A\&A, 128, 245 
Bucciantini, N., Amato, E., \& Del Zanna, L. 2005, A\&A, 434, 189 Camilo, F., Manchester, R. N., Lyne, A. G., et al. 2004, ApJ, 611, L25

Cash, W. 1979, ApJ, 228, 939

Cordes, J. M., \& Lazio, T. J. W. 2002, arXiv:astro-ph/0207156

de Jager, O. C., \& Djannati-Ataï, A. 2009, in Neutron Stars and Pulsars: 40 Years After Their Discovery, ed. W. Becker (Astrophys. Space Sci. Lib., Vol. 357; Berlin: Springer), 451

de Luca, A., Caraveo, P. A., Mereghetti, S., Negroni, M., \& Bignami, G. F. 2005, ApJ, 623, 1051

Duncan, A. R., Steward, R. T., Haynes, R. F., \& Jones, K. L. 1997, MNRAS, 287,722

Esposito, P., Tiengo, A., de Luca, A., \& Mattana, F. 2007, A\&A, 467, L45

Gaensler, B. M., Schulz, N. S., Kaspi, V. M., Pivovaroff, M. J., \& Becker, W. E. 2003, ApJ, 588, 441

Gaensler, B. M., \& Slane, P. O. 2006, ARA\&A, 44, 17

Gonzalez, M. E., Kaspi, V. M., Camilo, F., et al. 2005, ApJ, 630, 489

Griffith, M. R., \& Wright, A. E. 1993, AJ, 105, 1666

Helfand, D. J., Gotthelf, E. V., \& Halpern, J. P. 2001, ApJ, 556, 380

Hobbs, G., Lorimer, D. R., Lyne, A. G., \& Kramer, M. 2005, MNRAS, 360, 974

Horns, D., Aharonian, F., Hoffmann, A. I. D., \& Santangelo, A. 2007, Ap\&SS, 309,189

Johnson, S. P., \& Wang, Q. D. 2010, MNRAS, 408, 1216

Kargaltsev, O., Misanovic, Z., Pavlov, G. G., Wong, J. A., \& Garmire, G. P. 2008, ApJ, 684, 542

Kargaltsev, O., \& Pavlov, G. G. 2007, ApJ, 670, 655

Kargaltsev, O., \& Pavlov, G. G. 2008, in AIP Conf. Proc. 983, 40 Years of Pulsars: Millisecond Pulsars, Magnetars and More, ed. C. Bassa, Z. Wang, A. Cumming, \& V. M. Kaspi (Melville, NY: AIP), 171

Kargaltsev, O., \& Pavlov, G. G. 2010, in AIP Conf. Proc. 1248, X-Ray Astronomy 2009: Present Status, Multi-wavelength Approach and Future Perspectives, ed. A. Comastri, L. Angelini, \& M. Cappi (Melville, NY: AIP), 25

Kargaltsev, O., Pavlov, G. G., \& Garmire, G. P. 2007, ApJ, 660, 1413

Kargaltsev, O., Pavlov, G. G., \& Wong, J. A. 2009, ApJ, 690, 891

Kargaltsev, O. Y., Pavlov, G. G., Zavlin, V. E., \& Romani, R. W. 2005, ApJ, 625, 307

Kennel, C. F., \& Coroniti, F. V. 1984, ApJ, 283, 710

Komissarov, S., \& Lyubarsky, Y. 2004, Ap\&SS, 293, 107

Lemoine-Goumard, M., Zavlin, V. E., Grondin, M.-H., et al. 2011, A\&A, 533, 102

Linsley, J. 1975, in Proc. 14th ICRC, ed. K. Pinkau (Vol. 2; Munich: Max Planck Institute for Extraterrestrial Physics), 592
Maccacaro, T., Gioia, I. M., Wolter, A., Zamorani, G., \& Stocke, J. T. 1988, ApJ 326,680

Mignani, R. P., Shearer, A., De Luca, A., et al. 2011, A\&A, 533, 101

Mori, K., Kargaltsev, O., Pavlov, G., et al. 2008, in 37th COSPAR Scientific Assembly, Montreal, Canada, Symposium E, session 12, E12-0049-08, 2105

Mori, K., Tsunemi, H., Miyata, E., et al. 2001, in ASP Conf. Ser. 251, New Century of X-Ray Astronomy, ed. H. Inoue \& H. Kunieda (San Francisco, CA: ASP), 576

Pavlov, G. G., Bhattacharyya, S., \& Zavlin, V. E. 2010, ApJ, 715, 66

Pavlov, G. G., Kargaltsev, O., \& Brisken, W. F. 2008, ApJ, 675, 683

Pavlov, G. G., Sanwal, D., \& Zavlin, V. E. 2006, ApJ, 643, 1146

Pavlov, G. G., Shibanov, Y. A., Ventura, J., \& Zavlin, V. E. 1994, A\&A, 289, 837

Pavlov, G. G., Shibanov, Y. A., Zavlin, V. E., \& Meyer, R. D. 1995, in The Lives of the Neutron Stars, ed. M. A. Alpar, U. Kiziloglu, \& J. van Paradijs (NATO ASI Ser. C, Vol. 450; Dordrecht: Kluwer), 71

Pavlov, G. G., Teter, M. A., Kargaltsev, O., \& Sanwal, D. 2003, ApJ, 591, 1157

Pavlov, G. G., Zavlin, V. E., Sanwal, D., Burwitz, V., \& Garmire, G. P. 2001, ApJ, 552, L129

Pavlov, G. G., Zavlin, V. E., \& Trümper, J. 1999, ApJ, 511, L45

Pellizzoni, A., Pilia, M., Possenti, A., et al. 2009, ApJ, 695, L115

Renaud, M., Hoppe, S., Komin, N., et al. 2008, in AIP Conf. Proc. 1085, High Energy Gamma-ray Astronomy, ed. F. A. Aharonian, W. Hofmann, \& F. Rieger (Melville, NY: AIP), 285

Roberts, M. S. E., Gotthelf, E. V., Halpern, J. P., Brogan, C. L., \& Ransom, S. M. 2007, in Proc. 363 WE-Heraeus Seminar on Neutron Stars and Pulsars, ed. W. Becker \& H.-H. Huang (MPE Report 291; Garching: Max Planck Institute for Extraterrestrial Physics), 24

Romani, R. W., Ng, C.-Y., Dodson, R., \& Brisken, W. 2005, ApJ, 631, 480

Sanwal, D., Pavlov, G. G., Kargaltsev, O. Y., et al. 2002, in ASP Conf. Ser. 271, Neutron Stars in Supernova Remnants, ed. P. O. Slane \& B. M. Gaensler (San Francisco, CA: ASP), 353

Skrutskie, M. F., Cutri, R. M., Stiening, R., et al. 2006, AJ, 131, 1163

Tsunemi, H., Mori, K., Miyata, E., et al. 2001, ApJ, 554, 496

van der Swaluw, E. 2005, Adv. Space Res., 35, 1123

Yakovlev, D. G., \& Pethick, C. J. 2004, ARA\&A, 42, 169

Zacharias, N., Monet, D. G., Levine, S. E., et al. 2005, VizieR Online Data Catalog, 1297, 0

Zavlin, V. E. 2007, ApJ, 665, L143

Zavlin, V. E., \& Pavlov, G. G. 2004, ApJ, 616, 452

Zhu, W. W., Kaspi, V. M., McLaughlin, M. A., et al. 2011, ApJ, 734, 44 